\title{
Equivalent-neighbor percolation models in two dimensions: Crossover between mean-field and short-range behavior
}

\author{
Yunqing Ouyang, ${ }^{1,}{ }^{*}$ Youjin Deng, ${ }^{1, \dagger}$ and Henk W. J. Blöte ${ }^{2}$ \\ ${ }^{1}$ Hefei National Laboratory for Physical Sciences at Microscale, Department of Modern Physics, \\ University of Science and Technology of China, Hefei 230027, China \\ ${ }^{2}$ Lorentz Institute, Leiden University, P.O. Box 9506, 2300 RA Leiden, The Netherlands
}

(Received 16 August 2018; revised manuscript received 19 October 2018; published 3 December 2018)

\begin{abstract}
We investigate the influence of the range of interactions in the two-dimensional bond percolation model by means of Monte Carlo simulations. We locate the phase transitions for several interaction ranges, as expressed by the number $z$ of equivalent neighbors. We also consider the $z \rightarrow \infty$ limit, i.e., the complete graph case, where percolation bonds are allowed between each pair of sites, and the model becomes mean-field-like. All investigated models with finite $z$ are found to belong to the short-range universality class. There is no evidence of a tricritical point separating the short-range and long-range behavior, such as is known to occur for $q=3$ and $q=4$ Potts models. We determine the renormalization exponent describing a finite-range perturbation at the mean-field limit as $y_{r} \approx 2 / 3$. Its relevance confirms the continuous crossover from mean-field percolation universality to short-range percolation universality. For finite interaction ranges, we find approximate relations between the coordination numbers and the amplitudes of the leading correction terms as found in the finite-size scaling analysis.
\end{abstract}

DOI: 10.1103/PhysRevE.98.062101

\section{INTRODUCTION}

The range of the interactions plays an important role in phase transitions. Systems with pair interactions decaying with a negative power of the distance are found to display a variety of universality classes, depending on that power as well as on the dimensionality [1-3]. A different way to modify the range of the interactions is specified in the so-called equivalent-neighbor models, in which the pair interactions are constant up to a range $r$ and zero at larger distances. These models are referred to as medium-range models. The effects of such interactions were already investigated in several twodimensional model systems, including the Ising model [4], i.e., the $q=2$ Potts model, and the $q=3$ and $q=4$ Potts models [5]. In these models, the range $r$ contributes markedly to the irrelevant temperature field near the short-range critical fixed point, and thereby to the corrections to scaling.

In the limit of $r \rightarrow \infty$, the equivalent-neighbor models become mean-field-like, while for sufficiently small $r$ they fall in the short-range universality classes. Two different crossover scenarios are known between these two extremes. In the Ising case, the crossover along the critical line is uniform from the unstable mean-field (MF) limit to the short-range fixed point [4], so that all models with finite $r$ belong to the shortrange universality class. In contrast, the MF fixed point is stable in the cases of the $q=3$ and $q=4$ Potts models. For $q=3$ there is an intermediate tricritical point [5], while for $q \rightarrow 4$ the critical and tricritical points merge into a special fixed point [6] with a marginal operator. References [4,5] and

\footnotetext{
*oymj@mail.ustc.edu.cn

${ }^{\dagger}$ Corresponding author: yjdeng@ustc.edu.cn
}

references therein have shown that the exponent describing the scaling of the inverse range of interactions is, in general, not equal to 1 , as a naive real-space renormalization argument might suggest, and even changes sign as a function of $q$.

To provide a quantitative analysis concerning the scenario for the percolation model, we investigate the equivalentneighbor version of this model with a finite and variable interaction range $r$. The short-range percolation model has already been studied for a long time, some early literature appears in Refs. [7-9] and references therein. The present medium-range model is defined by the probability distribution of the percolation bonds, described by the partition sum

$$
Z_{\text {perc }}=\sum_{\mathcal{G}} p^{N_{\mathrm{b}}}(1-p)^{N_{\mathrm{e}}-N_{\mathrm{b}}},
$$

where the sum is on all graphs $G$ covering a number of edges of the square lattice. This number "bonds" is denoted $N_{\mathrm{b}}$ and can assume all values from 0 up to complete covering. Edges connect each site to all of its neighbors within the interaction range $r$. The interaction range is roughly specified by $z \approx \pi r^{2}$, where the coordination number $z$ is the number of equivalent-neighbors interacting with a site of the square lattice. Each edge has a probability $p$ to be covered by $\mathcal{G}$. The total number of edges, including those that are not occupied, is $N_{\mathrm{e}}$.

The analysis of the percolation model makes use of the connection between the percolation model and the $q=1$ Potts [10] model. To illustrate the equivalence, we consider the partition sum of the $q$-state Potts model

$$
Z_{\text {Potts }}=\sum_{s_{i}} e^{K \sum_{\langle i, j\rangle} \delta_{s_{i} s_{j}}},
$$


where the $s_{i}$, labeled by the site number, sit on a square lattice and assume the values $s_{i}=1,2, \cdots, q$. At high temperatures $T$ ( $K$ small), the spins tend to take random values and the model is disordered, whereas at low $T(K$ large, $>0)$ the model is ferromagnetic, the spins tend to be in the same state, even at large distances.

Kasteleyn and Fortuin [11] introduced bond variables between interacting neighbors, which can have two values: absent or present. Then it is possible to sum out the Potts variables in $Z_{\text {Potts }}$ and one obtains [11]

$$
Z_{\text {Potts }}=Z_{\mathrm{RC}}=\sum_{\mathcal{G}} u^{N_{\mathrm{b}}} q^{N_{\mathrm{c}}},
$$

where $u=e^{K}-1$, and the graph $\mathcal{G}$ represents the KasteleynFortuin bond variables. The sum is on all such graphs, as in the percolation model Eq. (1). The number of connected components (clusters) in $\mathcal{G}$ is denoted by $N_{\mathrm{c}}$. In the random-cluster representation [11] of the Potts model, the bond percolation model is obtained by taking the $q \rightarrow 1$ limit. Dividing out a trivial factor in Eq. (3) then leads to

$$
e^{-K N_{\mathrm{e}}} Z_{\mathrm{RC}}=Z_{\text {perc }},
$$

where $Z_{\text {perc }}$ is given by Eq. (1) with $p=u e^{-K}=u /(u+1)$.

We are now facing the question whether, for $q=1$, the MF to short-range crossover is uniform, like for $q=2$, or more like the $q>2$ Potts models. We investigate this question by simulating systems on $L \times L$ square lattices with periodic boundary conditions, for a sequence of finite sizes $L$, by means of Monte Carlo method [12] which remains efficient for systems with interactions of a long range. We sampled the size of the largest cluster, and the second and fourth moments of the cluster-size distribution. Thus, we obtained the percolation equivalent of the Potts magnetization moments and the Binder ratio, a dimensionless number related to the Binder cumulant [13], as explained in Sec. II. We also sampled the wrapping probabilities, also described in Sec. II. For $z=4$, the nearest-neighbor model, the critical point [7] and the universality class [14] are known. To achieve our analysis of the crossover between MF and short-range percolation, we stepwise increase the coordination number from $z=4$ towards infinity, meaning that each site can interact with all other sites, the mean-field or complete-graph case.

For each of these values $z>4$, the percolation threshold is determined from the simulation results. At the same time, we investigate whether the critical behavior still belongs to the short-range universality class. For this purpose it is necessary to simulate systems of sizes that are sufficiently large in comparison with the finite range of the interactions. We apply a finite-size scaling (FSS) analysis to a few different observables, to determine the nature of the crossover phenomena. Since the amplitudes of the corrections to scaling are a measure of the irrelevant fields, we may expect that the finite-size scaling analysis will provide some useful information.

The outline of the rest of this paper is as follows. In Sec. II we provide an overview of some known aspects of the two-dimensional percolation model, including the MF description. This section also describes the role of magnetic quantities, and defines the Binder ratio defined on the critical cluster-size distribution. It also includes the finite-size scaling of the Binder ratio and of the critical wrapping probabilities, taking into account the logarithmic factors in the corrections to scaling, which are caused by the presence of two degenerate irrelevant exponents. Section III explains the Monte Carlo simulation method, and describes the sampled quantities. In Sec. IV we present the results of the analysis of the wrapping probabilities, for the models with $4 \leqslant z \leqslant 60$. We verify the universality of these models, and determine the amplitudes of the leading corrections to scaling. Details of this analysis are given in the Appendix, together with an investigation of several models that do not obey the condition stated under Eq. (1), namely that the interacting neighbor sites fill a circle of a given radius $r$. Thus, one can purportedly distinguish the effects due the number $z$ of neighbors and their distances on the correction amplitudes.

Results for the Binder ratio are included in Sec. IV, for models with finite $z$ as well as in the MF limit $z \rightarrow \infty$. Special attention is given to the crossover between the MF fixed point and the short-range percolation model, for the case of the Binder ratio as well as for the temperature and magnetic exponents. Finally, Sec. IV includes a numerical determination of the crossover exponent of the MF model associated with a finite interaction range. The paper concludes with a discussion of the main results in Sec. V.

\section{EXISTING THEORY AND RESULTS}

\section{A. Percolation and magnetism}

We illustrate the role of the magnetism in percolation, using the context of the $q$-state Potts model. The Hamiltonian for that model, including an external field is

$$
\mathcal{H}=-K \sum_{\langle i, j\rangle} \delta\left(\sigma_{i}, \sigma_{j}\right)-H \sum_{k=1}^{N} \delta\left(\sigma_{k}, 1\right),
$$

where the magnetic field $H$ acts on Potts state 1 only. The interaction in Eq. (5) can be alternatively formulated in terms of $(q-1)$-dimensional Potts spin vectors, to reflect the full geometric symmetry. This is achieved by substituting

$$
\delta\left(\sigma_{i}, \sigma_{j}\right)=\frac{1}{q}\left[1+(q-1) \overrightarrow{e_{\sigma_{i}}} \cdot \overrightarrow{e_{\sigma_{j}}}\right],
$$

where $\vec{e}_{1}, \vec{e}_{2}, \cdots, \overrightarrow{e_{q}}$ are $q$ unit vectors pointing from the center to the corners of a regular hypertetrahedron in $q-1$ dimensions. Thus,

$$
\overrightarrow{e_{\sigma}} \cdot \overrightarrow{e_{\sigma^{\prime}}}=\frac{q \delta\left(\sigma, \sigma^{\prime}\right)-1}{q-1} .
$$

The magnetization density of an $N$-spin system is

$$
\vec{m} \equiv \sum_{i=1, N} \frac{\overrightarrow{e_{\sigma_{i}}}}{N}
$$

The magnetization of the system along direction 1 contains a contribution from the spins in state 1 , as well as contributions from the other $q-1$ states which are, according to Eq. (7), weighted with a factor $-1 /(q-1)$. This still allows for a zero "magnetization" of the $q \rightarrow 1$ random-cluster model in its the disordered phase. Since all Potts spins in a cluster are in the same state, and different clusters are in randomly distributed 
Potts states, the size of the largest cluster is a good measure of the Potts spontaneous magnetization.

Furthermore, the second and fourth magnetization moments can be expressed in terms of the random-cluster size distribution as

$$
\left\langle m^{2}\right\rangle=\left\langle\sum_{i} c_{i}^{2}\right\rangle
$$

and

$$
\left\langle m^{4}\right\rangle=\left\langle\frac{q+1}{q-1}\left(\sum_{i} c_{i}^{2}\right)^{2}-\frac{2}{q-1} \sum_{i} c_{i}^{4}\right\rangle,
$$

where $c_{\mathrm{i}}$ is the density of cluster $i$. Equations (9) and (10) enable the sampling of magnetic quantities in the Potts and random-cluster models without using actual Potts spins. While sampling of Eq. (10) for $q=1$ is obviously problematic, Eqs. (9) and (10) indicate that even powers of the magnetization scale as sums over the same powers of the cluster sizes.

\section{B. Finite-size scaling}

To provide a basis for our finite-size scaling analysis, we make use of the the renormalization formulation, with, in addition to the usual scaling fields, also a finite-size parameter $L$ [15-17]. We write down the scaling equation for the freeenergy density of a model as a function of the finite size $L$ and the scaling fields, namely, the relevant temperature field $t$, the relevant magnetic field $h$, and two irrelevant fields $u_{1}$ and $u_{2}$ :

$$
\begin{aligned}
f\left(t, h, u_{1}, u_{2}, L\right)= & f_{\mathrm{a}}\left(t, h, u_{1}, u_{2}\right) \\
& +b^{-d} f_{\mathrm{s}}\left(b^{y_{t}} t, b^{y_{h}} h, b^{y_{1}} u_{1}, b^{y_{2}} u_{2}, L / b\right),
\end{aligned}
$$

where $f_{\mathrm{a}}\left(t, h, u_{1}, u_{2}\right)$ is the analytic part of the free energy and $f_{\mathrm{s}}\left(t, h, u_{1}, u_{2}, L\right)$ is its singular part, and $b$ is the linear scale factor of the transformation. The prefactor $b^{-d}$ is due to rescaling of the $d$-dimensional volume. The temperature field $t$ and the magnetic scaling field $h$ are, in lowest order, proportional to the distance $T-T_{\mathrm{c}}$ to the critical temperature, and to the physical magnetic field $H$, respectively. For further details, see, e.g., Ref. [18]. The relevance of $t$ and $h$ means that the associated exponents $y_{t}$ and $y_{h}$ are positive, while the irrelevant exponents $y_{1}$ and $y_{2}$ are negative. In the present percolation models, the distance $p_{\mathrm{c}}-p$ plays, up to a modeldependent multiplicative constant, the role of the temperature field $t$. The substitution $b=L$ leads to the FSS relation for the free energy:

$$
\begin{aligned}
f\left(t, h, u_{1}, u_{2}, L\right)= & f_{\mathrm{a}}\left(t, h, u_{1}, u_{2}\right) \\
& +L^{-d} f_{\mathrm{s}}\left(L^{y_{t}} t, L^{y_{h}} h, L^{y_{1}} u_{1}, L^{y_{2}} u_{2}, 1\right) .
\end{aligned}
$$

The Binder ratio $Q$ is defined as

$$
Q(t, L)=\left\langle m^{2}\right\rangle^{2} /\left\langle m^{4}\right\rangle
$$

where $\left\langle m^{2}\right\rangle$ and $\left\langle m^{4}\right\rangle$ are the magnetization moments of order two and four, respectively:

$$
\begin{gathered}
\left\langle m^{2}\right\rangle=L^{-d}\left(\frac{\partial^{2} f}{\partial H^{2}}\right)_{H=0}, \\
\left\langle m^{4}\right\rangle=L^{-3 d}\left(\frac{\partial^{4} f}{\partial H^{4}}\right)_{H=0}+3 L^{-2 d}\left(\frac{\partial^{2} f}{\partial H^{2}}\right)_{H=0}^{2},
\end{gathered}
$$

where $H$ is the physical magnetic field. These equations follow by differentiation of $f \equiv \ln Z_{\text {Potts }}$ to $H$, where the $H$ dependence is specified by Eq. (5). The correspondence between the derivatives with respect to $h$ and $H$ is

$$
\frac{\partial^{2} f}{\partial H^{2}}=f^{(2)}\left(\frac{\partial h}{\partial H}\right)^{2}+\cdots
$$

and

$$
\frac{\partial^{4} f}{\partial H^{4}}=f^{(4)}\left(\frac{\partial h}{\partial H}\right)^{4}+2 f^{(2)} \frac{\partial h}{\partial H} \frac{\partial^{3} h}{\partial H^{3}}+\cdots .
$$

For the present we neglect additional terms due to the dependence of the other scaling fields on $H$. Furthermore, by differentiating $k$ times with respect to $h$, one obtains

$$
\begin{aligned}
f^{(k)}\left(t, h, u_{1}, u_{2}, L\right)= & L^{k y_{h}-d} f_{\mathrm{s}}^{(k)}\left(L^{y_{t}} t, L^{y_{h}} h, L^{y_{1}} u_{1}, L^{y_{2}} u_{2}, 1\right) \\
& +f_{\mathrm{a}}^{(k)}\left(t, h, u_{1}, u_{2}\right),
\end{aligned}
$$

and $\left\langle m^{2}\right\rangle$ can be explicitly expressed as

$$
\left\langle m^{2}\right\rangle=L^{2 y_{h}-2 d}\left(\frac{\partial h}{\partial H}\right)^{2}\left(f_{\mathrm{s}}^{(2)}+L^{d-2 y_{h}} f_{\mathrm{a}}^{(2)}\right)+\cdots .
$$

The leading term in $\left\langle m^{2}\right\rangle^{2}$ is of order $L^{4 y_{h}-4 d}$, as well as that in $\left\langle m^{4}\right\rangle$. The $L^{4 y_{h}-4 d}$ factors in $Q$ due to $\left\langle m^{2}\right\rangle^{2}$ and $\left\langle m^{4}\right\rangle$ cancel, and the $L^{d-2 y_{h}} f_{\mathrm{a}}^{(2)}$ term in $\left\langle m^{2}\right\rangle$ indicates that there should be a correction term in $Q$ that behaves as $L^{d-2 y_{h}}$. This analytic background term originates from the $h$ dependence of the analytic part $f_{\mathrm{a}}$ of the free energy.

Furthermore, the nonuniversal geometric factors $(\partial h / \partial H)^{4}$ in the numerator and denominator of $Q$ cancel, and $f_{\mathrm{s}}$ is a universal function, so that it follows that the resulting finite-size scaling equation for $Q$ is universal in the scaling limit.

Next, we return to Eq. (16) in which we have neglected a term $(\partial f / \partial t)\left(\partial^{2} t / \partial H^{2}\right)$. In general, one expects that the temperature scaling field includes a quadratic term in the physical magnetic field $H$ :

$$
t=\sum_{k} \tau_{k}\left(p-p_{\mathrm{c}}\right)^{k}+\rho H^{2}+\cdots .
$$

As a consequence, the $t$-dependence of Eq. (12) leads to an additional correction term proportional to $L^{y_{t}-2 d}$ in Eq. (19). The relative scale of this correction is $L^{y_{t}-2 y_{h}}$. It similarly affects $\left\langle m^{4}\right\rangle$ and $Q$. Combining the universal part and the additional corrections, we obtain

$$
\begin{aligned}
Q\left(t, u_{1}, u_{2}, L\right)= & Q\left(L^{y_{t}} t, L^{y_{1}} u_{1}, L^{y_{2}} u_{2}, 1\right) \\
& +b_{3} L^{d-2 y_{h}}+b_{4} L^{y_{t}-2 y_{h}}+\cdots .
\end{aligned}
$$

The analytic part of the free energy does not contribute to the wrapping probabilities $R_{\mathrm{w}}$, which are, like $Q$, 
dimensionless. The finite-size scaling formula is

$$
R_{\mathrm{w}}\left(t, u_{1}, u_{2}, L\right)=R_{\mathrm{w}}\left(L^{y_{t}} t, L^{y_{1}} u_{1}, L^{y_{2}} u_{2}, 1\right) .
$$

\section{Degenerate irrelevant exponents}

In the $d=2$ Potts model, there is an exponent -2 for general $q$. For a numerical justification, see Ref. [19]. In addition, the second thermal exponent of the Potts model depends on $q$ and assumes the value -2 in the percolation case $q=1$ [14]. Under these circumstances, the the irrelevant exponents become degenerate and the corresponding scaling fields could couple with each other [20]. Suppose that, in differential form, one has the following scaling equations for the irrelevant fields:

$$
\begin{gathered}
\frac{d u_{\mathrm{i}}(l)}{d l}=y_{\mathrm{i}} u_{\mathrm{i}}(l), \\
\frac{d u_{1}(l)}{d l}=y_{\mathrm{i}}\left[u_{1}(l)+\alpha u_{\mathrm{i}}(l)\right],
\end{gathered}
$$

where $l$ parametrizes the renormalization flow such that the linear scale factor is $b=e^{l}$. The Roman indices " $\mathrm{i}$ " and "l" stand for "irrelevant" and "logarithmic," respectively. The solution is [20]

$$
\begin{gathered}
u_{\mathrm{i}}(l)=b^{y_{\mathrm{i}}} u_{\mathrm{i}}(0), \\
u_{1}(l)=b^{y_{\mathrm{i}}}\left[u_{1}(0)+\alpha u_{\mathrm{i}} \ln (b)\right],
\end{gathered}
$$

which shows that the rescaling of the irrelevant fields as used in Eqs. (21) and (22) has to be modified by adding a logarithmic factor. To avoid confusion, the notation of the two irrelevant fields is changed accordingly. After substitution of the correct rescaling of $u_{\mathrm{i}}$ and $u_{1}$ into the scaling equations Eqs. (21) and (22), setting $b=L$, and Taylor expansion, one obtains

$$
\begin{gathered}
Q(t, L)=Q+\sum_{j=1}^{\infty} q_{j} t^{j} L^{j y_{t}}+b_{\mathrm{i}} L^{y_{\mathrm{i}}}+b_{1} \ln (L) L^{y_{\mathrm{i}}} \\
+b_{3} L^{d-2 y_{h}}+b_{4} L^{y_{t}-2 y_{h}}+\cdots \\
R_{\mathrm{w}}(t, L)=R_{\mathrm{w}}+\sum_{j=1}^{\infty} a_{j} t^{j} L^{j y_{t}}+b_{\mathrm{i}} L^{y_{\mathrm{i}}} \\
+b_{1} \ln (L) L^{y_{\mathrm{i}}}+\cdots
\end{gathered}
$$

These formulas describe the deviations from universal values $Q$ of the Binder ratio and $R_{\mathrm{w}}$ of the wrapping probabilities in finite systems near the critical point, thus providing the basis for the FSS analysis of the numerical data.

Since, close to the critical point, $p-p_{\mathrm{c}} \propto t$, Eqs. (27) and (28) describe the dependence of these two dimensionless quantities on the bond probability $p$ and system size $L$. The polynomial function in $p$ takes into account the effect caused by the deviation of $p$ from the critical bond probability $p_{\mathrm{c}}$, while the critical finite-size effect is described by the correction terms $b_{\mathrm{i}} L^{y_{\mathrm{i}}}+b_{1} \ln (L) L^{y_{\mathrm{i}}}$ due to irrelevant scaling fields. Both $b_{\mathrm{i}}$ and $b_{1}$ are analytic functions of $u_{i}$ and $u_{j}$. In addition there is an analytic background term with amplitude $b_{3}$ for the Binder ratio.
For models with small coordination number $z$, deviations from isotropy are relatively large and will be taken into consideration, which could be observable in the correction terms, $b_{\mathrm{i}} L^{-2}$ and $b_{1} \ln (L) L^{-2}$.

For percolation in two dimensions, several critical exponents are exactly known [14]. The leading and subleading thermal renormalization exponents are $y_{t}=1 / v=3 / 4$, and $y_{\mathrm{i}}=-2$, respectively; the leading critical exponent in the magnetic sector is $y_{h}=91 / 48$, and the exponent of the correction due to the analytic background term is thus $-43 / 24$.

\section{Mean-field percolation}

In mean-field (MF) percolation, every site can connect to any other site. The behavior of this model is well known [21-24]. We summarize the derivation of the temperature and magnetic exponents. These exponents do also apply in high-dimensional systems [25] with short-range couplings. In the MF case, one has $z=L^{2}$, and the edges of the lattice thus form a complete graph. The critical bond probability $p$ is thus inversely related to the total number of sites $L^{2}$. It makes therefore sense to work with the integrated (i) bond probability $p_{\mathrm{i}} \equiv p L^{2}$.

To construct the self-consistency equation for this model, we again use the framework of the $q$-state Potts model. The Hamiltonian for this model on the complete graph is, analogous to Eq. (5),

$$
\mathcal{H}=-\frac{K}{N} \sum_{i<j}^{N} \delta\left(\sigma_{i}, \sigma_{j}\right)-H \sum_{k=1}^{N} \delta\left(\sigma_{k}, 1\right) .
$$

We expect that, at high temperatures, i.e., small $K, \vec{m}=0$ in the thermodynamic limit, and that, at low temperatures, a spontaneous magnetization exists in the direction of one of the unit vectors mentioned, for example, direction 1 . We thus formulate our analysis in terms of that component $m=|\vec{m}|$ of the magnetization density.

The canonical probability $P_{1}$ that a spin assumes state 1 is

$$
P_{1}=\frac{e^{K N_{1} / N+H}}{e^{K N_{1} / N+H}+(q-1) e^{K\left(N-N_{1}\right) /[N(q-1)]}},
$$

where $N_{1} / N=[1+(q-1) m] / q$ is the density of spins in state 1 . Substitution of $N_{1}$ yields

$$
P_{1}=\frac{e^{K m+H}}{e^{K m+H}+q-1} .
$$

Expansion of this expression to first order in $H$ and second order in $m$ leads asymptotically to

$$
P_{1}=\frac{1}{q}+(q-1)\left[\frac{K m+H}{q}-\frac{K^{2} m^{2}(2-q)}{2 q}\right] .
$$

The requirement of self-consistency imposes that $m=$ $\left(q P_{1}-1\right) /(q-1)$ or

$$
P_{1}=\frac{1}{q}+m \frac{q-1}{q} .
$$

Substitution in Eq. (32) leads to a quadratic equation in $m$,

$$
\frac{(1-t)^{2}(2-q) m^{2}}{q}+2 t m-2 H=0
$$


where we have substituted the temperature-like variable $t \equiv$ $1-K$. We have divided out a factor $q-1$, and the interpretation of this equation for $q=1$ has to be done with some care. The variable $m$ loses its meaning as a magnetization, but it keeps its meaning as the density of the largest cluster in the ordered phase, also in the $q \rightarrow 1$ limit of the random-cluster model. In this limit, Eq. (34) reduces, for small $t$, to

$$
m^{2}+2 t m-2 H=0,
$$

which provides a description of the critical region. The critical point is located at $t=H=0$. For $H=0$, one has $m \propto-t$, which determines the critical exponent $\beta=1$. For $t=0$, one has $m \propto \sqrt{H}$, so that $\delta=2$. Differentiation to $H$ yields the susceptibility-like quantity $\chi \propto t^{-1}$ at $H=0$, and therefore $\gamma=1$.

The exponents $\beta, \gamma, \delta$ can be expressed in effective renormalization exponents and the dimensionality $d$ of the system as

$$
\beta=\frac{d-y_{h}^{*}}{y_{t}^{*}}, \quad \gamma=\frac{2 y_{h}^{*}-d}{y_{t}^{*}}, \quad \delta=\frac{y_{h}^{*}}{d-y_{h}^{*}},
$$

which yields

$$
y_{h}^{*}=2 d / 3, \quad y_{t}^{*}=d / 3 .
$$

The asterisks in $y_{h}^{*}$ and $y_{t}^{*}$ may require some explanation. The actual renormalization exponents $y_{t}$ and $y_{h}$ do not satisfy the relation in Eqs. (36) without asterisks. The reason is that MF models violate "hyperscaling" relations: scaling relations that involve the dimensionality $d$ [26,27]. The renormalization exponents with an asterisk describe the rescaling of the observables that are conjugate to the scaling fields. The notation with asterisks serves to reproduce the usual dependence of the critical exponents on $y_{t}$ and $y_{h}$, as expressed by Eqs. (36). In our case, $d=2, y_{h}^{*}=\frac{4}{3}$, and $y_{t}^{*}=$ $\frac{2}{3}$. The critical exponent $\alpha=\left(2 y_{t}^{*}-d\right) / y_{t}^{*}=-1$ describes a specific-heat-like quantity in the percolation model [28], and the exponent $\beta$ describes the size of the largest percolation cluster as a function of $p-p_{\mathrm{c}}$. The magnetic exponent also determines the finite-size scaling of the largest cluster at $t=0$ as asymptotically proportional to $L_{h}^{y_{h}^{*}}[24]$.

\section{METHODS}

\section{A. Simulation algorithms}

The simulation of models with many interacting neighbors tends to be time consuming, if all these bonds are individually taken into account. This problem is circumvented by means of an algorithm [12], employed earlier in the analysis of the medium-range Potts model with $q=2$ [4], and with $q=3$ and 4 [5]. The computer time needed depends only weakly on the number of interactions per site. The application of this medium-range algorithm to the percolation case $q=1$ is straightforward.

\section{B. Sampled quantities}

The wrapping probabilities $R_{\mathrm{w}}$ investigated in this paper are specified as $R_{\mathrm{b}}, R_{\mathrm{e}}$ and $R_{1}$, defined as follows. Let $x$ and $y$ specify the main axes of the square lattice. Then, the probability $R_{1}$ counts the events that a configuration of percolation bonds wraps the periodic box such that it connects to itself along the $x$ direction, but not along the $y$ direction. For better statistical accuracy, we count every configuration which percolates in one direction, but not in both directions, and divide the total percolations by a factor of $2 ; R_{\mathrm{b}}$ is for simultaneous wrapping in both directions; $R_{\mathrm{e}}$ is for wrapping in at least one direction [29]. The relevant factors that may be related to their universal values are the number of couplings between a central site and its neighbors within the interaction range, the boundary conditions and the shape of the system. To what extent the microscopic couplings between the central site and its different neighbors, i.e., the interaction range, are important is the present subject of investigation.

In this analysis, it is very useful that the universal values of the wrapping probabilities are known for models with short-range interactions and periodic boundary conditions in a square geometry. These are $R_{\mathrm{b}}=0.351642855, R_{\mathrm{e}}=$ 0.690473725 , and $R_{1}=0.169415435$ [30-32].

The Binder ratio $Q$ of the $q$-state Potts model is defined by Eq. (13) on the basis of the distribution of the magnetization density $m$. Using the random-cluster representation, the magnetization moments, and thus $Q$, can be determined from the random-cluster size distribution via Eqs. (9) and (10). The latter quantity is divergent for $q \rightarrow 1$, but for a scalable cluster-size distribution, we may instead just define a universal ratio for the percolation case by using the Ising-like definition with $q=2$ :

$$
Q=\frac{\left\langle\sum_{i} c_{i}^{2}\right\rangle^{2}}{\left\langle 3\left(\sum_{i} c_{i}^{2}\right)^{2}-2 \sum_{i} c_{i}^{4}\right\rangle} .
$$

This definition was used during the simulations. Its universal value for two-dimensional percolation in a square periodic geometry has been estimated as $Q=0.87048(5)[33,34]$.

\section{RESULTS}

\section{A. Analysis of the wrapping probabilities}

We simulated finite systems with medium-range interaction and sampled the three kinds of wrapping probabilities mentioned earlier, $R_{\mathrm{b}}, R_{\mathrm{e}}$, and $R_{1}$. In this work we restrict ourselves to systems with square symmetry, so that we can analyze our data in the light of the known universal wrapping probabilities [31] of the short-range model. The coupling strengths for all the $z$ interacting neighbors are the same.

The simulations took place for roughly 30 different system sizes, ranging from small ones, with $L$ slightly larger than the interaction range, up to $L=2048$ in some cases. The larger systems serve to approximate the asymptotic behavior in the thermodynamic limit, including the determination of the critical point and the renormalization exponents $y_{t}$ and $y_{h}$. The corrections to scaling in the $R_{\mathrm{w}}(p, L)$ data (w $=\mathrm{b}, \mathrm{e}$, or 1$)$ are relatively small for large $L$, so that the corresponding error sources are suppressed. However, relatively small systems are needed to obtain accurate estimations of the amplitudes of $b_{\mathrm{i}}$ and $b_{1}$ of the corrections to scaling. The typical lengths of the simulations varied from millions of samples for the largest systems to billions for the smallest ones.

First, we performed simulations of systems whose interaction regions are almost circular. These interactions are fully 

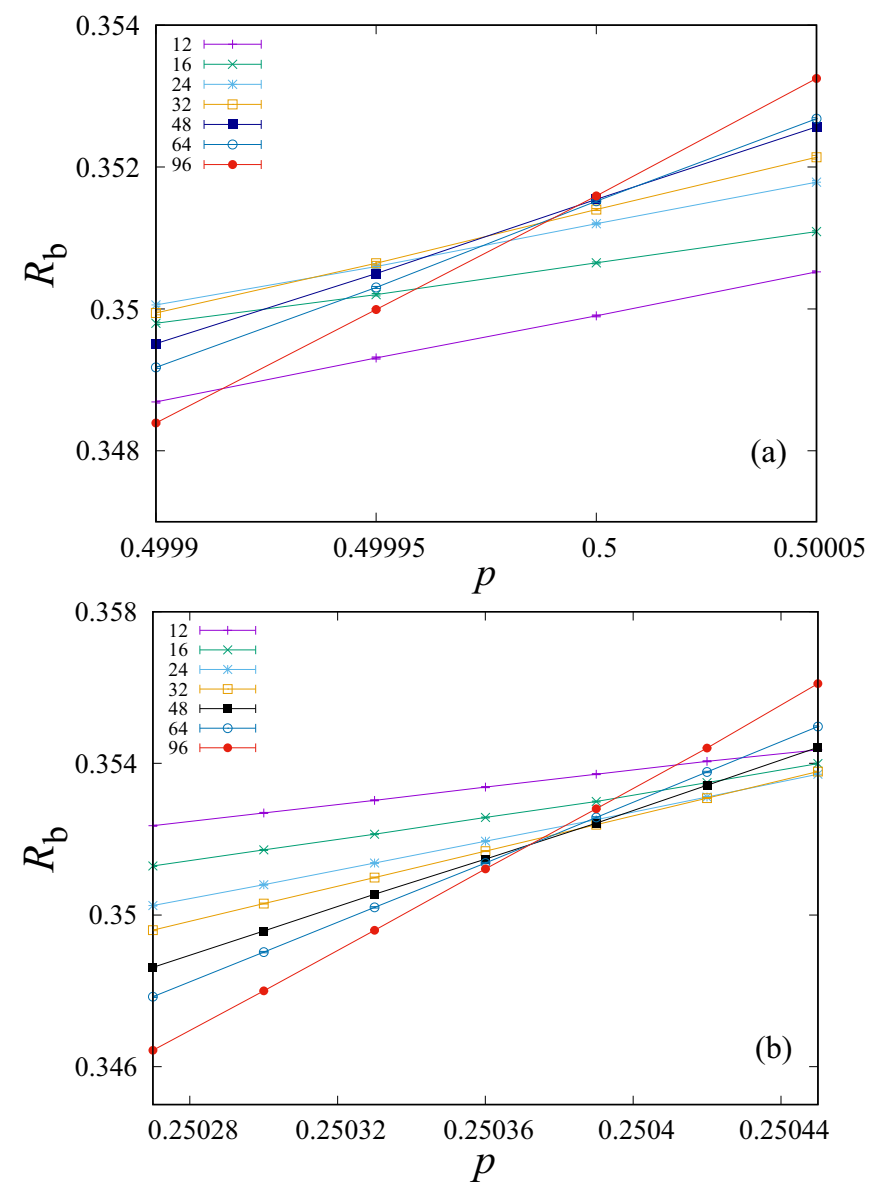

FIG. 1. $R_{\mathrm{b}}(L, p)$ versus $p$ plots for the 4- and 8-neighbor models. Figure (a) applies to $z=4$ and (b) to $z=8$. The system sizes are in the range from 12 to 96 as shown in the figure. Larger systems correspond to steeper curves.

determined by the range $r$, such each site couples with all $z$ neighbors within that range. The corresponding values of $z$ studied are $z=4,8,12,20,28,36,48,60,224,1224$, and 4016. In the Appendix, we shall investigate the additional influence of the range of these interactions, by relaxing the rule that the $z$ interacting neighbors fill a circle. We also check for effects due to fourfold deviations of the sets of neighbors from isotropy.

\section{Tests of universality of the wrapping probabilities}

On the basis of Eq. (28) we expect that plots of the wrapping probabilities versus $p$ for different $L$ display intersections, at least if $a_{1}$ is nonzero. For models in the short-range percolation universality class, these intersections should, for large $L$, converge to the universal value of $R_{\mathrm{w}}$. The numerical results for the wrapping probabilities can thus be used as a test whether models with $z>4$ do still belong to the shortrange universality class. This is illustrated in Fig. 1 for the case of $R_{\mathrm{b}}$. While the corresponding curves for $R_{\mathrm{e}}$ display a similar behavior, those for $R_{1}(p, L)$ appear to be different, because the amplitude $a_{1}$ is zero or very small. Self-duality imposes that $a_{1}=0$ for the $z=4$ model. Thus, intersections are not useful for the determination of the critical point from

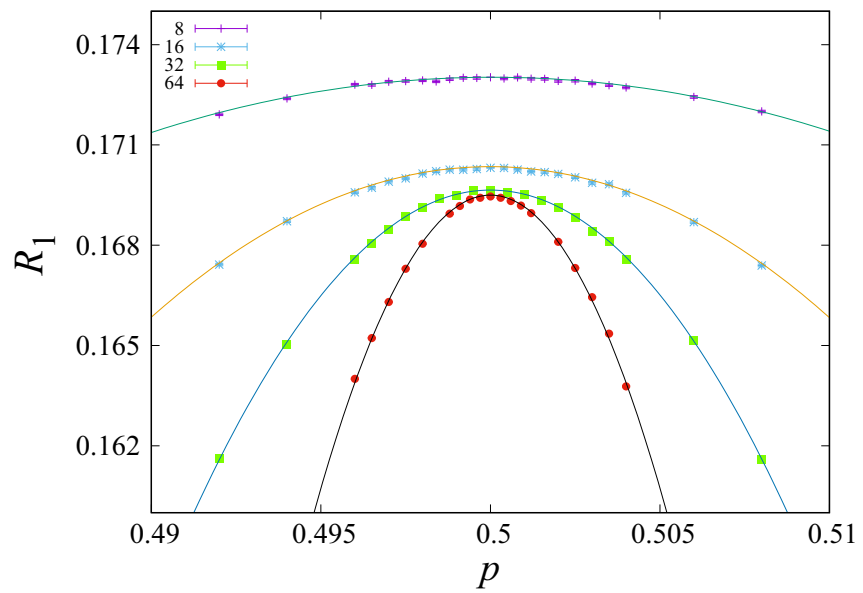

FIG. 2. $R_{1}(L)$ vs. $p$ for $L=8,16,32$, and 64 for the $z=4$ model. Symmetry of this figure is imposed by the self-duality [7] for $z=4$.

$R_{1}(p, L)$, as shown in Fig. 2. The amplitude of the quadratic term is nonzero, as illustrated by the non-monotonic behavior of $R_{1}$. We fitted finite-size wrapping data by

$$
\begin{aligned}
R_{\mathrm{w}}(p, L)= & R_{\mathrm{w}}+\sum_{k} a_{k}\left(p-p_{\mathrm{c}}\right)^{k} L^{k y_{t}}+b_{\mathrm{i}} L^{-2} \\
& +b_{1} \ln (L) L^{-2}+b_{3} L^{-3},
\end{aligned}
$$

which also takes into account a subleading correction of order $L^{-3}$. Integer differences between subleading exponents may be expected in general [35]. Since the simulations were performed in a rather narrow neighborhood of the critical point, higher-order terms in $p-p_{\mathrm{c}}$ rapidly become unimportant.

The fits for $R_{\mathrm{b}}$ and $R_{\mathrm{e}}$ yielded consistent estimates of $p_{\mathrm{c}}$. These values of $p_{\mathrm{c}}$ were kept fixed in the fits for $R_{1}$. The resulting estimates for $R_{\mathrm{b}}, R_{\mathrm{e}}$, and $R_{1}$ are in a fairly accurate agreement with the short-range universal values, thus confirming that the investigated models with $z>4$ still obey short-range universality. These results are listed in Table I.

\section{Universality of the critical exponents}

After confirming that the universal values of the wrapping probabilities of $R_{\mathrm{b}}$ and $R_{\mathrm{e}}$ still apply for the values of $z$ investigated, we fixed them in the fit formula while leaving $y_{t}$ as a free parameter in Eq. (28). Again, the results are in agreement with the short-range universal value $y_{t}=3 / 4$. They are included in Table I.

As another test, we determine the magnetic renormalization exponent $y_{h}$. We first fixed the wrapping probabilities and $y_{t}$ at their universal values, and obtained improved estimates of the critical points $p_{\mathrm{c}}$ of the models with $z>4$. The average density $c_{1}$ of the largest cluster was then fitted by

$$
\begin{aligned}
c_{1}(p, L)= & L^{y_{h}-2}\left[\sum_{k=0,1,2, \cdots} g_{k}\left(p-p_{\mathrm{c}}\right)^{k} L^{k y_{t}}\right. \\
& \left.+b_{\mathrm{i}} L^{-2}+b_{1} \ln (L) L^{-2}+b_{3} L^{-3}+\cdots\right],
\end{aligned}
$$


TABLE I. Universal parameters as estimated for models with different coordination numbers $z$.

\begin{tabular}{|c|c|c|c|c|c|}
\hline$z$ & $R_{\mathrm{b}}$ & $R_{\mathrm{e}}$ & $R_{1}$ & $y_{t}$ & $y_{h}$ \\
\hline 4 & $0.351643(6)$ & $0.690464(5)$ & $0.169413(4)$ & $0.749(2)$ & $1.8958(2)$ \\
\hline 8 & $0.351641(6)$ & $0.690479(5)$ & $0.169416(5)$ & $0.748(2)$ & $1.896(1)$ \\
\hline 12 & $0.351649(4)$ & $0.69037(3)$ & $0.169411(3)$ & $0.747(3)$ & $1.895(2)$ \\
\hline 20 & $0.35167(2)$ & $0.69053(2)$ & $0.169409(3)$ & $0.748(2)$ & $1.894(3)$ \\
\hline 28 & $0.35165(3)$ & $0.69053(5)$ & $0.169407(5)$ & $0.747(3)$ & $1.895(2)$ \\
\hline 36 & $0.35163(3)$ & $0.69051(3)$ & $0.169409(4)$ & $0.748(3)$ & $1.897(2)$ \\
\hline 48 & $0.35167(3)$ & $0.69052(4)$ & $0.169405(4)$ & $0.746(4)$ & $1.896(1)$ \\
\hline 60 & $0.3517(1)$ & $0.6904(1)$ & $0.16941(2)$ & $0.747(2)$ & $1.895(2)$ \\
\hline exact & 0.351642855 & 0.690473725 & 0.169415435 & 0.75 & 1.89583333 \\
\hline
\end{tabular}

with $p_{\mathrm{c}}$ as determined from the wrapping probabilities. The results appear to be in a good agreement with the short-range universal value $y_{h}=91 / 48$ [14]. The results are included in Table I. Finally, we performed a test if there is a contribution from the second magnetic exponent $y_{h 2}=19 / 48$ [14] to the wrapping probabilities. If so, one may expect corrections with a relative magnitude in the order $L^{y_{h 2}-y_{h}}=L^{-3 / 2}$. However, no evidence for such a correction was found.

\section{Determination of the correction amplitudes}

Having sufficient confidence in the validity of the shortrange universal parameters, we kept them fixed in the fits according to Eq. (28), with the purpose to find the best estimates of the critical points and the amplitudes of the correction terms as a function of $z$.

We find that the correction amplitudes of the wrapping probabilities are approximately proportional to $z^{2}$, and thus to the squared area within the interaction range. This is illustrated in Fig. 3 for the wrapping probabilities $R_{\mathrm{b}}$ and $R_{\mathrm{e}}$. The amplitudes $b_{\mathrm{i}}$ and $b_{1}$, appear to become small between $z=4$ and $z=8$, suggestive of a short-range fixed point in that neighborhood. The overall finite-size scaling effect due to the three correction terms displays a change of sign between $z=4$ and 8, as can, for example, be seen in Fig. 1. A more

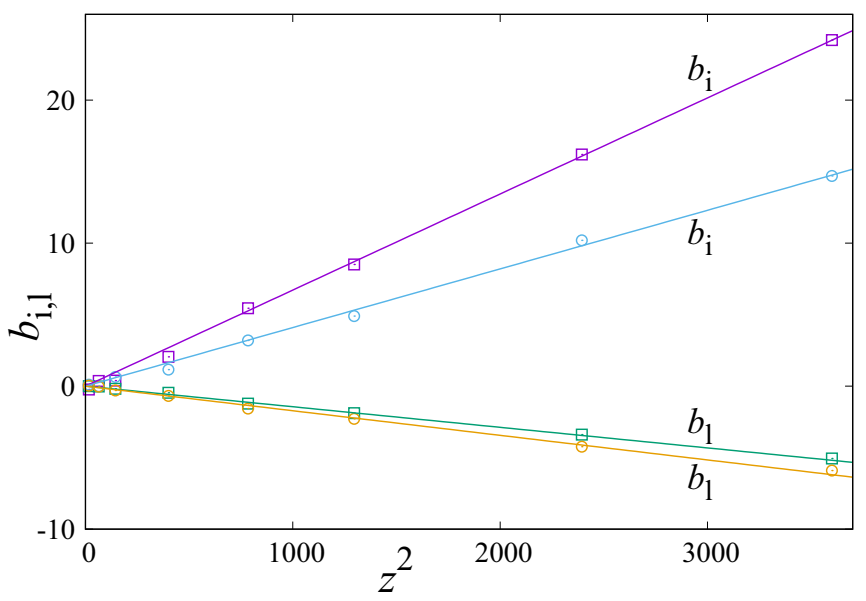

FIG. 3. Correction amplitudes $b_{\mathrm{i}}$ and $b_{1}$ in the wrapping probabilities $R_{\mathrm{b}}(L, p)$ (squares) and $R_{\mathrm{e}}(L, p)$ (circles), as a function of $z^{2}$. Data are shown for models with up to $z=60$ neighbors. detailed analysis, including tabulated data for the correction amplitudes and the critical points, is deferred to the Appendix.

\section{B. FSS analysis for the Binder ratio $Q$}

\section{Binder ratio of models with finite $z$}

The behavior of $Q(p, L)$, as defined by Eq. (38), of the nearest-neighbor model $(z=4)$ is illustrated in Fig. 4 for several system sizes as a function of $p$. The intersections of the lines are seen to converge to the critical point [7] at $p_{\mathrm{c}}=0.5$. For small $L$, the combined finite-size corrections are positive at the critical point. In contrast, they are negative for the $z \geqslant 8$ models, as seen, e.g., in Fig. 5. The sign changes of the leading correction terms are analogous to those of the wrapping probabilities.

Just as in the case of the wrapping probabilities, we analyzed several models with finite coordination numbers up to $z=60$. The increasing distance to the short-range fixed point manifests itself as $z$ increases. Figure 5 shows that strong crossover effects already occur in the 60-neighbor model: the intersections between the curves with small $L$ are located at relatively small values of $Q$, but for larger $L$ they still approach the universal value $[33,34]$ of the short-range model.

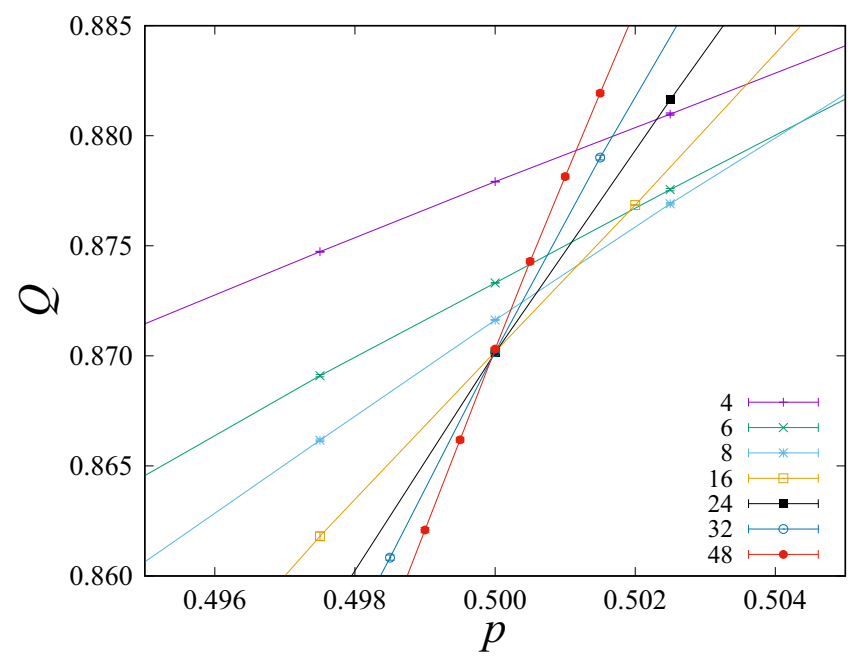

FIG. 4. Binder ratio $Q$ of the bond-percolation model with nearest-neighbor interaction vs. bond probability $p$, for several system sizes. In the present $z=4$ case, the corrections are positive for small $L$ and negative for large $L$. 


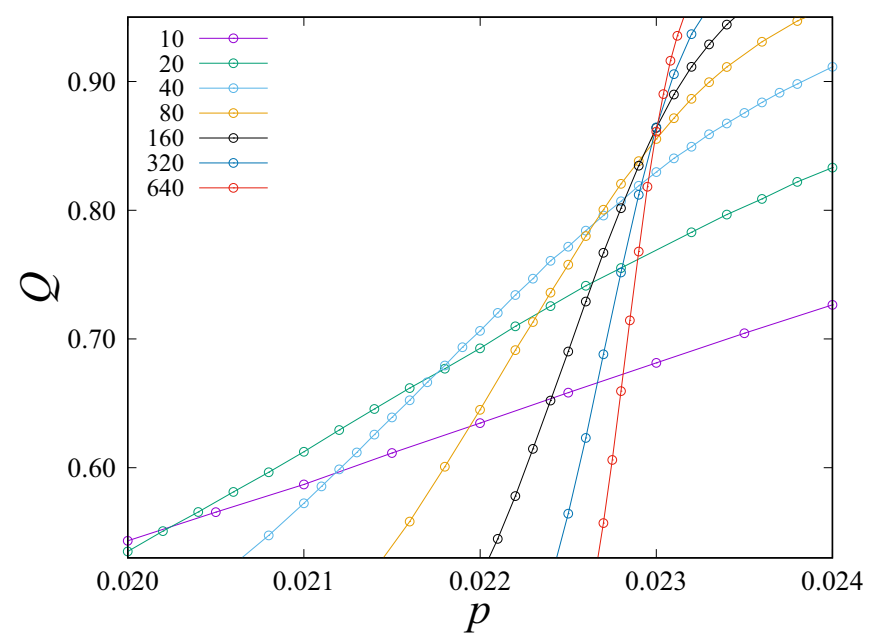

FIG. 5. Binder ratio $Q(p, L)$ of the bond-percolation model with 60 equivalent neighbors vs. bond probability $p$. The figure shows data for several system sizes as indicated in the figure. It displays that strong corrections to scaling occur in the 60-neighbor model. Larger systems correspond to steeper curves.

As described in Sec. II B 1 , these $Q$ data are expected to behave as

$$
\begin{aligned}
Q(p, L)= & Q\left(p_{\mathrm{c}}, \infty\right)+\sum_{k} q_{k}\left(p-p_{\mathrm{c}}\right)^{k} L^{k y_{t}}+b_{\mathrm{i}} L^{-2} \\
& +b_{1} \ln (L) L^{-2}+b_{3} L^{d-2 y_{h}}+b_{4} L^{y_{t}-2 y_{h}}+\cdots
\end{aligned}
$$

For models in the short-range percolation universality class, we have $y_{t}=3 / 4$ and $y_{h}=91 / 48$ [14]. Since $p_{c}$ can be determined more accurately from the wrapping probabilities than from the Binder ratio, we fixed the critical points at the values determined from the wrapping probabilities. Leastsquares fits were applied in rather narrow $p$-intervals and included only few $p$-dependent terms. In the case $z=4$, where the corrections appear to be small and we know the exact critical point [7], we could determine the universal value of the Binder ratio as $Q=0.87057(2)$, slightly higher than a previous result $Q=0.87048(5)$ [33,34]. After checking that the Binder ratios of the $z>4$ models were consistent with this value, the newer result for $Q$ was used as a fixed parameter in the fits for the $z>4$ models. The amplitudes of the correction terms are found to increase rapidly with $z$. This is illustrated in Fig. 6, which shows that the relation between $b_{\mathrm{i}}$ and $z$ is approximately described by a quadratic form, as shown by the parabola that figure. This behavior is very different from that of the amplitudes in the wrapping probabilities. The numerical results for the correction amplitudes in the Binder ratios are listed in the Appendix.

\section{Binder ratio of the complete graph}

As shown in Table II, the critical point $p_{\mathrm{c}}$ is a decreasing function of $z$. For large $z$, the critical point behaves approximately as $1 / z$. According to the MF solution of the percolation model in Sec. IIC, the critical value of the integrated Potts coupling is equal to 1 . Since the bond probability is, in first order, equal to the Potts coupling (see Sec. I), we must

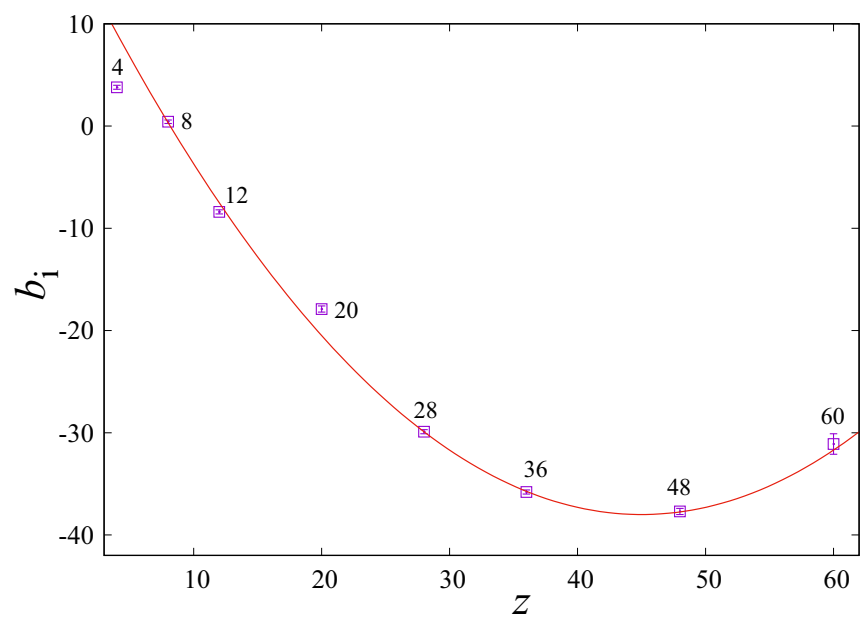

FIG. 6. Correction to scaling amplitude $b_{\mathrm{i}}$ in the Binder ratio $Q$ vs. number of interacting neighbors $z$. These data, which are taken from Table VIII, are approximately described by the parabola shown in the figure.

have $N p_{\mathrm{c}} \rightarrow 1$ for a system of $N \rightarrow \infty$ sites. Therefore, in simulations of finite systems we simply use a bond probability $p=p_{\mathrm{i}} / L^{2}$, so that the critical point of the integrated probability lies at $p_{\mathrm{i}}=1$.

Since the wrapping probabilities cease to be meaningful on the complete graph, we restrict ourselves here to the Binder ratio. Results for $Q(p, L)$ of the complete graph are shown in Fig. 7. As the system size $L$ goes up, the intersections of the $Q(p, L)$ curves are seen to converge to a value in agreement with the exact critical point $p_{\mathrm{i}}=1$. The corresponding critical value of $Q$ for the complete graph is clearly different from the universal short-range percolation value.

Fits based on the form of Eq. (41) were applied, but using the integrated bond probability $p_{\mathrm{i}}$ instead, with the critical point fixed at $p_{\mathrm{i}, \mathrm{c}}=1$. The leading correction exponent was found to be close to $-2 / 3$, without a logarithm, in agreement with recent work of Huang et al. [36]. The results of the fit of the $Q$ data for the complete graph are clearly inconsistent with the universal exponents of the finite- $z$ models. After fixing $y_{t}=2 / 3$ and $y_{h}=4 / 3$, in accordance with Eqs. (36), and leaving $Q$ as a free parameter, we obtained satisfactory fits. The results, and further details, are included in Table VIII.

\section{Crossover phenomena}

\section{Crossover of the Binder ratio}

To demonstrate the crossover between the MF and shortrange models, we first construct a data collapse by means of a $z$-dependent rescaling of the finite size. We define $L_{\mathrm{r}} \equiv$ $L / b(z)$ with $b(4)=b(8)=1$ and $b(z)$ for $z>8$ chosen such that the $Q\left(p_{\mathrm{c}}, L\right)$ versus $L_{\mathrm{r}}$ curves collapse for $L \geqslant 8$. It is not possible to include the $z=4$ data in this collapse because the corrections have the opposite sign. These results are shown in Fig. 8. Also shown in this figure is the finite-size dependence of the $Q$ results for the critical MF model versus the inverse finite size. Our use of $1 / L_{\mathrm{cg}}$ and our definition of $L_{\mathrm{r}}$ for the horizontal scale is tuned such that the finite-size data for $Q$ of the MF model just match with those of the finite- $z$ models. 
TABLE II. Finite-size scaling of $R_{\mathrm{b}}$. The coordination number $z$, the critical point $p_{\mathrm{c}}$, the minimum system size $L_{\mathrm{min}}$ of the fits, and the amplitudes $b_{\mathrm{i}}, b_{1}$, and $b_{3}$ of the correction terms due to the irrelevant fields are listed. The number of degrees of freedom is defined as the number of data points minus the number of fitted parameters and may be compared with the residual $\chi^{2}$. The value of $L_{\text {min }}$ is chosen such that the residual, as well as the fitted parameters, become at most weakly dependent on the minimum system size used.

\begin{tabular}{|c|c|c|c|c|c|c|c|}
\hline$z$ & $p_{\mathrm{c}}$ & $L_{\min }$ & $b_{\mathrm{i}}$ & $b_{1}$ & $b_{3}$ & $\chi^{2}$ & $d_{\mathrm{f}}$ \\
\hline \multirow{2}{*}{4} & $1 / 2$ & 9 & $-0.261(10)$ & $0.006(3)$ & $-0.13(4)$ & 37.5 & 40 \\
\hline & $1 / 2$ & 10 & $-0.261(15)$ & $0.006(2)$ & $-0.13(6)$ & 37.1 & 38 \\
\hline \multirow{2}{*}{8} & $0.25036843(9)$ & 8 & $0.34(2)$ & $-0.022(7)$ & $-0.28(7)$ & 40.7 & 47 \\
\hline & $0.25036847(9)$ & 10 & $0.31(5)$ & $-0.012(13)$ & $-0.16(16)$ & 36.5 & 45 \\
\hline \multirow{2}{*}{12} & $0.15222023(4)$ & 7 & $0.39(1)$ & $-0.187(5)$ & $-0.56(5)$ & 74.5 & 73 \\
\hline & $0.15222022(4)$ & 8 & $0.42(3)$ & $-0.194(9)$ & $-0.7(1)$ & 73.6 & 72 \\
\hline \multirow{2}{*}{20} & $0.08415091(3)$ & 12 & $2.06(6)$ & $-0.47(2)$ & $-4.0(2)$ & 30.1 & 45 \\
\hline & $0.08415093(3)$ & 13 & $1.98(8)$ & $-0.45(2)$ & $-3.7(4)$ & 27.7 & 44 \\
\hline \multirow{2}{*}{28} & $0.05584923(3)$ & 12 & $5.2(1)$ & $-1.16(3)$ & $-14.2(4)$ & 18 & 26 \\
\hline & $0.05584925(3)$ & 14 & $5.0(2)$ & $-1.11(4)$ & $-13.3(7)$ & 15.5 & 25 \\
\hline \multirow{2}{*}{36} & $0.04169607(2)$ & 10 & $8.51(6)$ & $-1.89(2)$ & $-27.3(2)$ & 68.6 & 60 \\
\hline & $0.04169608(2)$ & 11 & $8.40(8)$ & $-1.86(2)$ & $-26.8(3)$ & 64 & 59 \\
\hline \multirow{2}{*}{48} & $0.02974268(1)$ & 14 & $16.2(1)$ & $-3.39(3)$ & $-63.6(5)$ & 46.2 & 35 \\
\hline & $0.02974268(1)$ & 16 & $16.2(2)$ & $-3.38(5)$ & $-63(1)$ & 45 & 33 \\
\hline \multirow{2}{*}{60} & $0.02301189(2)$ & 16 & $24.2(8)$ & $-5.1(1)$ & $-98(4)$ & 38.6 & 37 \\
\hline & $0.02301191(2)$ & 20 & $22.6(9)$ & $-4.5(2)$ & $-96(5)$ & 36.6 & 36 \\
\hline
\end{tabular}

One observes that these data for $Q$ span the whole range between the universal values of the MF and the short-range model. There is no sign of a possible intermediate multicritical point when $z$ increases. The collapse of the other curves on that for $z=8$ involves a rescaling factor $b(z)$ that satisfies the relation

$$
\begin{aligned}
b_{\mathrm{i}}(8) & L^{-2}+b_{1}(8) \ln (L) L^{-2}+b_{3}(8) L^{d-2 y_{h}} \\
= & b_{\mathrm{i}}(z) b(z)^{2} L^{-2}+b_{1}(z) b(z)^{2} \ln [L / b(z)] L^{-2} \\
& +b_{3}(z) b(z)^{2 y_{h}-d} L^{d-2 y_{h}} .
\end{aligned}
$$

Among these correction terms, $b_{3} L^{d-2 y_{h}}$ is the dominant one for large $L$ and asymptotically determines $b(z)$ in that limit as $b(z) \simeq\left[b_{3}(8) / b_{3}(z)\right]^{1 /\left(2 y_{h}-d\right)}$.

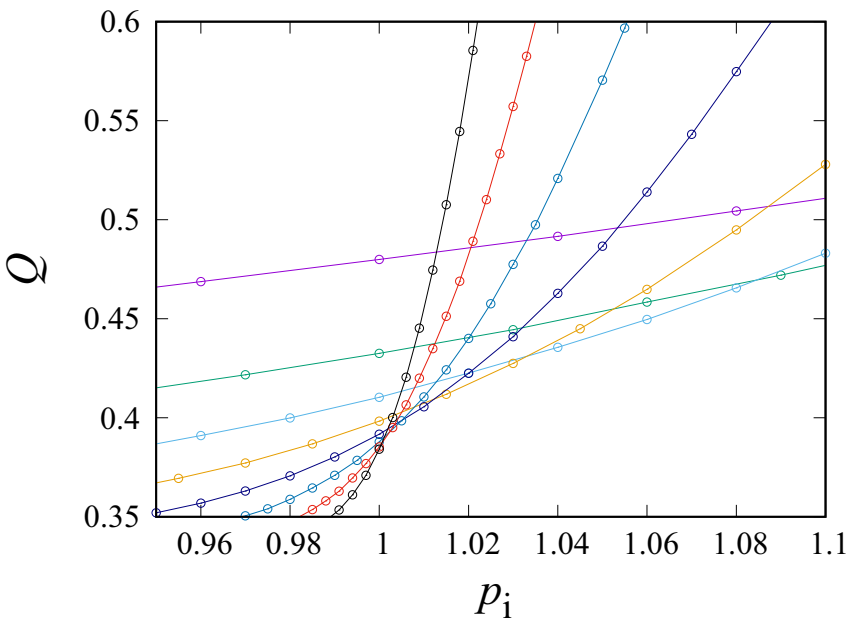

FIG. 7. Binder ratio $Q(p, L)$ of the MF percolation model vs. integrated bond probability $p_{\mathrm{i}}$, for system sizes $L=4,8,16,32$, $64,128,256$, and 512. Larger system sizes correspond with steeper curves. The intersections of these curves approach $p_{\mathrm{i}}=1$ for the larger systems.

\section{Crossover of the effective temperature exponent}

As another demonstration of the crossover phenomenon, we analyze the first derivative of $Q(p, L)$ with respect to the bond probability $p$ at criticality [5]. From Eq. (41) one expects that, at the transition point, it behaves as

$$
\frac{\ln (d Q / d p)}{\ln (L)}=y_{t}+\frac{\ln \left(a_{1}\right)+\cdots}{\ln (L)} .
$$

The data for $d Q / d p$, were obtained from fits to the $Q$ versus $p$ simulation results and by numerical differentiation. Since

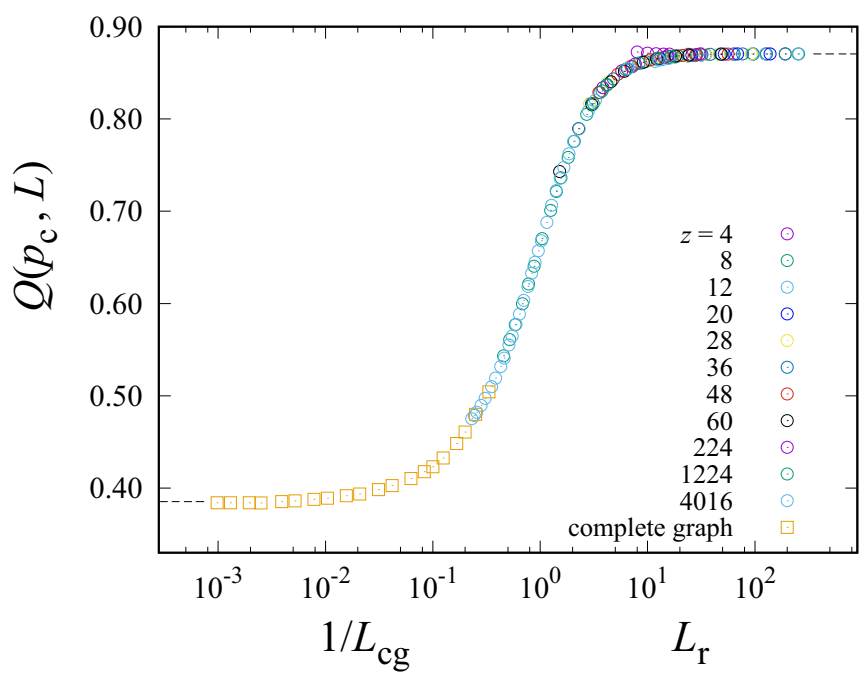

FIG. 8. $Q\left(p_{\mathrm{c}}, L\right)$ vs. rescaled size $L_{\mathrm{r}}$ for $z<\infty$. The rescaling factor $b(z)$ in $L_{\mathrm{r}}$ is defined in the text for the finite $z$ models. The data for $z=4$ cannot be collapsed similarly because the corrections have the opposite sign. The leftmost data apply to the largest $z$. The figure includes data for the complete graph (cg), for which we adopt the inverse finite size $1 / L_{\mathrm{cg}}$ as the finite-size parameter. The asymptotic values of $Q$ for $L \rightarrow \infty$ are 0.87057 and 0.38126 , respectively, marked by dashed lines in the figure. 


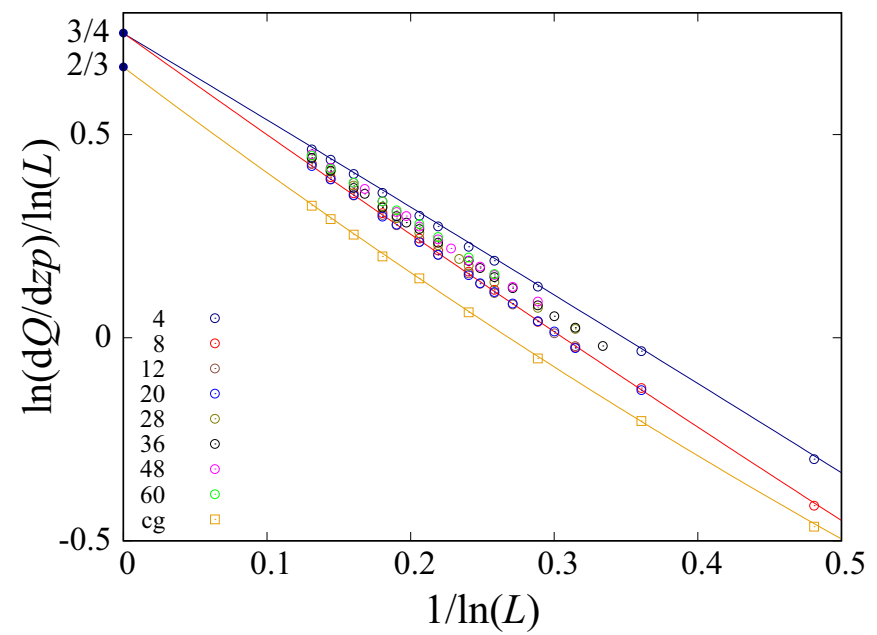

FIG. 9. Finite-size dependence of the derivative of the Binder ratio $Q$ to the integrated bond probability $z p$. Different symbols indicate percolation models with different coordination numbers, as listed in the figure. These results show an approximate data collapse for the finite- $z$ systems. There is a clear difference with the data for the complete graph, or the MF limit, which are also shown. The quantity plotted along the vertical scale is defined in the text, and chosen such that the large- $L$ data should converge to the temperature exponent $y_{t}$ which is $3 / 4$ for short-range percolation, and 2/3 for MF percolation. The data points for $z=4$, and $z=12$, and for the MF model are connected by curves which are also intended to guide the eye to the limiting value at $L=\infty$ on the vertical scale.

$p_{\mathrm{c}}$ is roughly proportional to $1 / z$, we include a factor $1 / z$ in the derivative of $Q$ to $p$. A comparison of the result, shown in Fig. 9, with similar analyses for the $q=3$ and 4 Potts models in Ref. [5] illustrates the different dependencies of these models on the range of the interactions.

The $z$-dependence of the effective temperature exponent can also be illustrated with the quantity

$$
\left.\left.y_{t, \mathrm{eff}}(L) \equiv \ln [\{d Q(p, 2 L) / d p)\} /\{d Q(p, L) / d p)\right\}\right]_{p=p_{\mathrm{c}}} / \ln 2,
$$

which is shown in Fig. 10 as a function of $L^{y_{\mathrm{i}}}$.

\section{Crossover of the effective magnetic exponent}

The determination of the magnetic exponent from the scaling behavior of the density of the largest cluster, as described in Sec. IV A 2 for the finite- $z$ models, was also done for the complete graph. Figure 11 shows the combined results on logarithmic scales. While the finite- $z$ data display a good data collapse for not too small $L$, the results for the complete graph fall on a line with a different slope.

We also quantitatively analyzed the scaling behavior of the density of the largest cluster in the MF limit at the critical point $p_{\mathrm{i}, \mathrm{c}}=1$. Analogous to Eq. (40), it is expected to behave as $c_{1}\left(p_{\mathrm{i}, \mathrm{c}}, L\right)=g_{0} L^{y_{h}-2}+\cdots$. Corresponding fits yielded the estimate $y_{h}=1.3333(2)$, in a good agreement with $y_{h}=4 / 3$ as given in Sec. II C for the mean-field model.

The exponent $y_{h}$ can be directly estimated by comparing two different system sizes $L_{1}$ and $L_{2}$, as $y_{h} \approx \ln \left[c_{1}\left(p_{\mathrm{i}, \mathrm{c}}, L_{1}\right) / c_{1}\left(p_{\mathrm{i}, \mathrm{c}}, L_{2}\right)\right] / \ln \left(L_{1} / L_{2}\right)+2$. Figure 12 displays the results for the models with finite $z$ as well as for the complete graph.

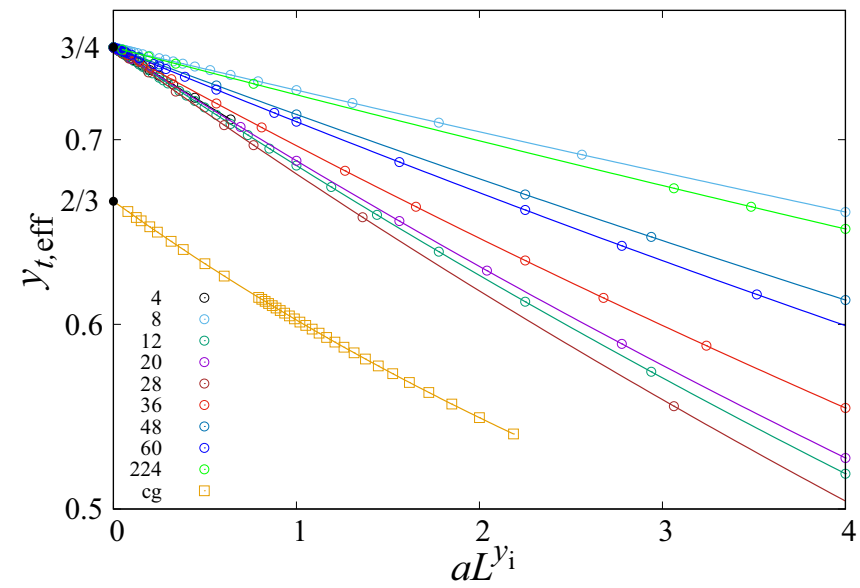

FIG. 10. Effective temperature exponent $y_{t, \text { eff }}$, defined in the text, as a function of $a L^{y_{i}}$. To fit the curves of the various interaction ranges in the same figure, the amplitude $a$ is arbitrarily chosen as 8 for the mean-field limit, and as $z^{2}$ in the other cases. The finite- $z$ data are bracketed by the lines for $z=8$ (above) and $z=28$ (below). The horizontal scale used the values $y_{\mathrm{i}}=-2 / 3$ and -2 , respectively. The data for each of the models show satisfactory large- $L$ convergence to the expected values of the temperature exponent $y_{t}$, namely $3 / 4$ for the short-range models, and $2 / 3$ for the MF case.

\section{The crossover exponent at the MF fixed point}

The critical points, in terms of the integrated bond probabilities $p_{\mathrm{i}, \mathrm{c}}=z p_{\mathrm{c}}$, are shown in Fig. 13 versus $z^{-1 / 2}$. The latter quantity describes the inverse range of the interactions when $z$ is large. Among the renormalization exponents describing the flow in this diagram, the one at the MF fixed point along the critical line is still undetermined. This exponent, purportedly describing the effect of interactions with a large but less than infinite range, is denoted $y_{r}$. To determine this exponent, we analyze the shape of the critical line $p_{\mathrm{i}, \mathrm{c}}$. For

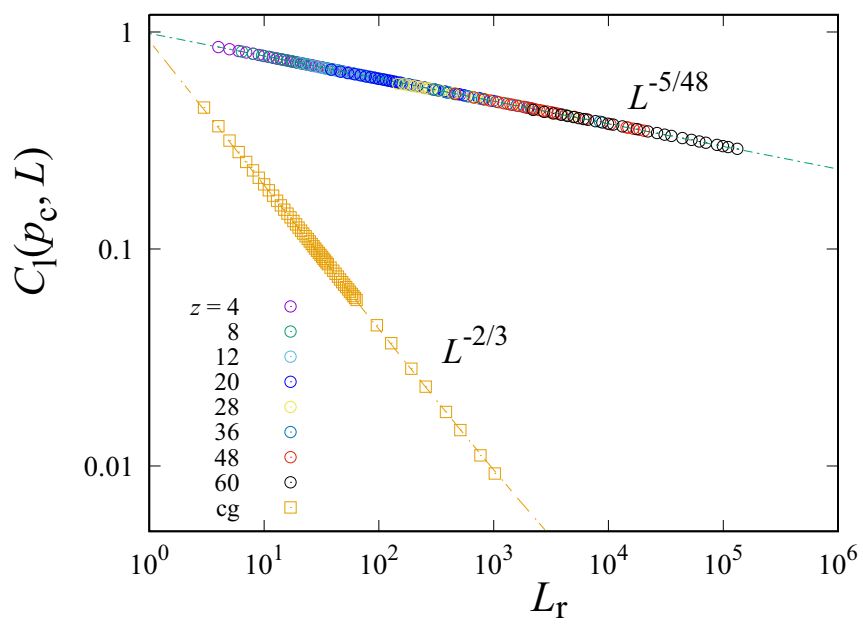

FIG. 11. Critical density of the largest cluster versus rescaled finite-size $L_{\mathrm{r}}$. For the complete graph we define $L_{\mathrm{r}}=L$, and for finite $z$, we define $L_{\mathrm{r}}$ as $L$ times a $z$-dependent constant, chosen such that the large- $L$ data collapse onto the $z=4$ data. The large- $z$ data dominate the right-hand part of the upper line. Logarithmic scales are used to display the power-law nature of these results. Straight lines are shown according to the exactly known exponents. 


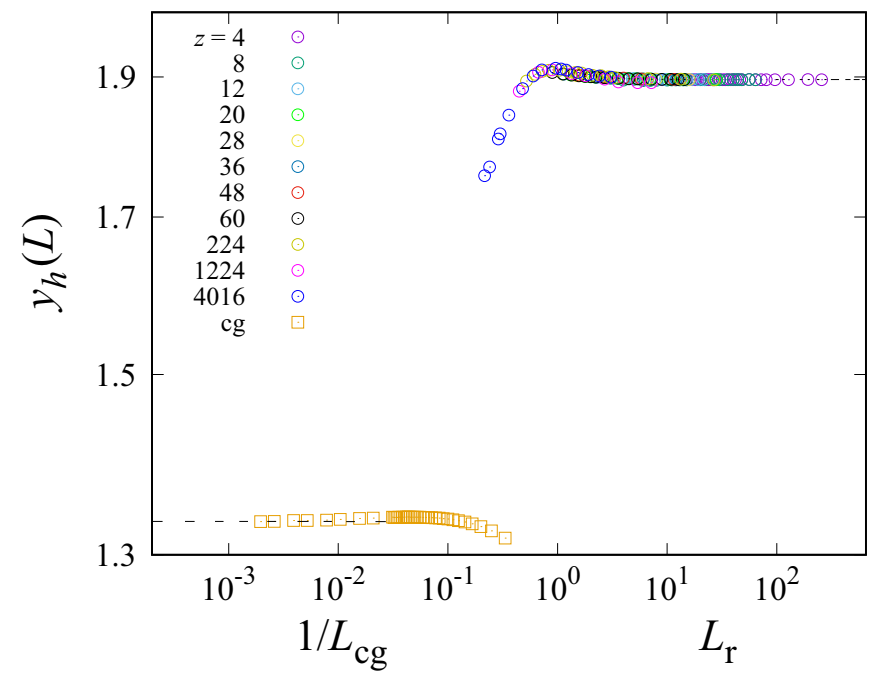

FIG. 12. Finite-size estimates of the magnetic exponent $y_{h}$ versus rescaled finite size, as determined from the largest-cluster sizes at the percolation threshold. The large- $z$ data occupy the left-hand side of the upper curve. The data for the complete graph use the $1 / L_{\mathrm{cg}}$ scale, and the finite- $z$ data use the $L_{\mathrm{r}}$ scale, obtained by rescaling $L$ for $z>8$ such that the data collapse as well as possible onto results for smaller $z$.

this purpose we parametrize the neighborhood of the MF fixed point by a temperature variable $v_{t} \equiv p_{\mathrm{i}}-1$, and an inverse range parameter $v_{r} \equiv z^{-1 / 2}$. Under a renormalization transformation by a scale factor $b$, these variables become

$$
v_{t}(b)=b^{y_{t}} v_{t}, \quad v_{r}(b)=b^{y_{r}} v_{r} .
$$

It is essential that we allow that the inverse range renormalizes with a new exponent $y_{r}$ that is different from the naive value 1. It follows from Eq. (45) that the critical line in the vicinity of the MF fixed point satisfies $v_{t}(b) \propto v_{r}(b)^{y_{t} / y_{r}}$,

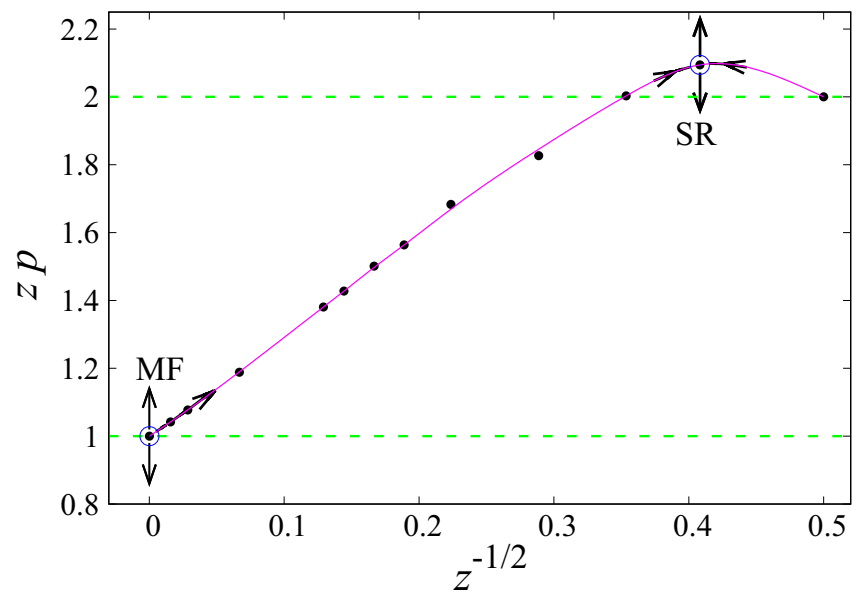

FIG. 13. Integrated critical bond probability $z p_{\mathrm{c}}$ versus $z^{-1 / 2}$ diagram. Shown are the critical points (black circles) and a smoothed critical line on that basis. The fixed points are shown as blue circles. The MF fixed point is located at $z=\infty$. Since the corrections to scaling become small between $L=4$ and 8 , we place the short-range (SR) fixed point at $z=6$. We illustrate this point using the exactly known critical point of the triangular lattice bond percolation model. Also shown is the renormalization flow near the fixed points. so that $z p_{\mathrm{c}}-1 \propto\left(z^{-1 / 2}\right)^{y_{t} / y_{r}}$ in Fig. 13. The approximate linearity of the critical line near the MF fixed point therefore suggests that $y_{r}=y_{t}=2 / 3$. This is further confirmed by a fit of $\left(a_{1}+a_{2} z^{-1 / 2}\right)\left(z^{-1 / 2}\right)^{1 / \phi}$ to $z p_{\mathrm{c}}-1$, for $z=224,1224$, and 4016, using our Monte Carlo results $p_{\mathrm{c}}=0.0053050415$ (33), 0.000880188 (90), and 0.0002594722 (11), respectively. This yielded a result for the crossover exponent as $\phi^{-1}=y_{t} / y_{r}=1.006$ (7).

\section{CONCLUSION}

The numerical analyses of several models with coordination numbers up to $z=60$ agree accurately with the universal constants $Q, R_{\mathrm{b}}, R_{\mathrm{e}}$, and $R_{1}$. These models fit the theory of a short-range fixed point describing all percolation models with a limited range of interaction.

Near the short-range fixed point, the amplitudes of the corrections to scaling in the wrapping probabilities were found to be approximately proportional to the square of the number of neighbors $z$, or the fourth power of the interaction range $r$. These corrections are governed by the irrelevant exponent $y_{i}=-2$. Scaling then indicates that, in a vicinity of the shortrange fixed point, the interaction range scales as $r^{\prime}=b^{-1 / 2} r$ under a scale reduction with a factor $b$.

In this work, we have also included a few models with larger $z$ up to $z=4016$, corresponding to a range $r=35.78$. While the results of the numerical analysis of the large- $z$ models are consistent with the universal constants mentioned, their accuracy falls off when $z$ increases. Using a rescaling of the finite-size parameter, a satisfactory data collapse of the critical $Q$ versus $L$ curves could be obtained, including the large $z$ models. These data illustrate the crossover of $Q$ from the complete graph to the short-range universal value, as shown in Fig. 8.

The analysis of $d Q / d p$ in Sec. IV C 1 indicates that the models with finite $z$ behave in accordance with the short-range temperature exponent $y_{t}=3 / 4$, again obeying short-range universality. The behavior of the complete graph model is found to be different, with $y_{t}=2 / 3$ instead, as predicted by the theory.

These results indicate that the crossover from MF to shortrange percolation takes place in a uniform way, with the MF limit acting as a fixed point that is unstable along the critical line in the $p$ versus $z$ diagram, as illustrated in Fig. 13. There is no sign of an intermediate higher critical fixed point along this line. Such an additional fixed point would moreover imply that the MF fixed point is stable in the direction of the critical line, contradicting the result $y_{r}=2 / 3$ in Sec. IV C 4. The renormalization flow thus leads directly to the short-range fixed point, which is stable in the range direction. This situation is similar to that found for the range-dependent crossover of the Ising model [4].

\section{ACKNOWLEDGMENTS}

Y.D. thanks the Ministry of Science and Technology of China for Grant No. 2016YFA0301604 and the National Natural Science Foundation of China for Grant No. 11625522. 
TABLE III. Finite-size scaling analysis of $R_{\mathrm{e}}$. The quantities listed are the same as those in Table II.

\begin{tabular}{|c|c|c|c|c|c|c|c|}
\hline$z$ & $p_{\mathrm{c}}$ & $L_{\min }$ & $b_{\mathrm{i}}$ & $b_{1}$ & $b_{3}$ & $\chi^{2}$ & $d_{\mathrm{f}}$ \\
\hline \multirow{2}{*}{4} & $1 / 2$ & 9 & $0.115(10)$ & $0.030(3)$ & $0.15(3)$ & 43 & 40 \\
\hline & $1 / 2$ & 10 & $0.120(14)$ & $0.029(4)$ & $0.12(5)$ & 43 & 38 \\
\hline \multirow{2}{*}{8} & $0.25036863(10)$ & 9 & $-0.11(4)$ & $-0.009(11)$ & $0.28(12)$ & 61.1 & 41 \\
\hline & $0.25036858(10)$ & 10 & $-0.03(6)$ & $-0.030(16)$ & $0.0(2)$ & 57.7 & 40 \\
\hline \multirow{2}{*}{12} & $0.15222030(7)$ & 10 & $0.64(7)$ & $-0.30(2)$ & $0.1(2)$ & 57.1 & 65 \\
\hline & $0.1522204(1)$ & 11 & $0.7(1)$ & $-0.30(3)$ & $-0.1(5)$ & 52.2 & 64 \\
\hline \multirow{2}{*}{20} & $0.08415101(3)$ & 12 & $1.05(6)$ & $-0.66(2)$ & $-0.7(2)$ & 36.3 & 51 \\
\hline & $0.08415102(3)$ & 13 & $1.04(7)$ & $-0.65(2)$ & $-0.7(3)$ & 36.2 & 50 \\
\hline \multirow{2}{*}{28} & $0.05584935(4)$ & 12 & $2.7(2)$ & $-1.45(4)$ & $-2.5(7)$ & 14.7 & 28 \\
\hline & $0.05584933(5)$ & 14 & $3.0(2)$ & $-1.51(6)$ & $-4(1)$ & 13 & 27 \\
\hline \multirow{2}{*}{36} & $0.04169613(2)$ & 11 & $4.8(1)$ & $-2.27(3)$ & $-6.3(5)$ & 46.1 & 50 \\
\hline & $0.04169613(2)$ & 12 & $4.9(2)$ & $-2.28(4)$ & $-6.6(7)$ & 45.9 & 49 \\
\hline \multirow{2}{*}{48} & $0.02974271(1)$ & 14 & $10.1(2)$ & $-4.20(4)$ & $-19.4(8)$ & 44 & 34 \\
\hline & $0.02974271(1)$ & 16 & $10.2(3)$ & $-4.23(7)$ & $-20(1)$ & 41.6 & 32 \\
\hline \multirow{2}{*}{60} & $0.02301187(3)$ & 16 & $14.7(6)$ & $-5.9(1)$ & $-20(3)$ & 30.8 & 41 \\
\hline & $0.02301189(3)$ & 20 & 13(1) & $-5.6(2)$ & $-19(6)$ & 28.1 & 40 \\
\hline
\end{tabular}

TABLE IV. Finite-size scaling analysis of $R_{1}$. These data are not suitable for determining the critical point $p_{\mathrm{c}}$ and we keep $p_{\mathrm{c}}$ in the fitting formula at fixed values adopted from $R_{\mathrm{b}}$.

\begin{tabular}{|c|c|c|c|c|c|c|c|}
\hline$z$ & $p_{\mathrm{c}}$ & $L_{\min }$ & $b_{\mathrm{i}}$ & $b_{1}$ & $b_{3}$ & $\chi^{2}$ & $d_{\mathrm{f}}$ \\
\hline \multirow{2}{*}{4} & $1 / 2$ & 9 & $0.188(5)$ & $0.012(1)$ & $0.14(2)$ & 41 & 40 \\
\hline & $1 / 2$ & 10 & $0.189(7)$ & $0.012(2)$ & $0.13(3)$ & 40.8 & 38 \\
\hline \multirow{2}{*}{8} & 0.2503685 & 9 & $-0.184(17)$ & $-0.005(2)$ & $0.14(6)$ & 66 & 46 \\
\hline & 0.2503685 & 10 & $-0.145(24)$ & $-0.016(7)$ & $0.00(9)$ & 62 & 45 \\
\hline \multirow{2}{*}{12} & 0.1522203 & 10 & $0.17(2)$ & $-0.069(6)$ & $0.1(1)$ & 68.7 & 71 \\
\hline & 0.1522203 & 11 & $0.19(3)$ & $-0.073(7)$ & $0.0(1)$ & 68.2 & 70 \\
\hline \multirow{2}{*}{20} & 0.0841509 & 12 & $-0.44(2)$ & $-0.111(7)$ & $1.4(1)$ & 47.9 & 60 \\
\hline & 0.0841509 & 13 & $-0.41(3)$ & $-0.119(8)$ & $1.3(1)$ & 44.6 & 59 \\
\hline \multirow{2}{*}{28} & 0.0558493 & 12 & $-1.10(6)$ & $-0.18(2)$ & $5.4(3)$ & 24.4 & 29 \\
\hline & 0.0558493 & 14 & $-0.91(9)$ & $-0.23(2)$ & $4.3(4)$ & 15.2 & 28 \\
\hline \multirow{2}{*}{36} & 0.04169608 & 11 & $-1.72(5)$ & $-0.22(1)$ & $10.0(2)$ & 63.4 & 61 \\
\hline & 0.04169608 & 12 & $-1.63(7)$ & $-0.25(2)$ & $9.6(3)$ & 59.3 & 60 \\
\hline \multirow{2}{*}{48} & 0.02974268 & 12 & $-2.95(5)$ & $-0.43(1)$ & $21.7(2)$ & 45.2 & 36 \\
\hline & 0.02974268 & 14 & $-2.95(7)$ & $-0.43(2)$ & 21.7(3) & 44.5 & 34 \\
\hline \multirow{2}{*}{60} & 0.0230119 & 16 & $-5.2(2)$ & $-0.43(5)$ & 43(1) & 19.6 & 37 \\
\hline & 0.0230119 & 20 & $-5.3(5)$ & $-0.4(1)$ & $43(3)$ & 19.5 & 36 \\
\hline
\end{tabular}

TABLE V. Finite-size-scaling analysis of $R_{\mathrm{b}}$ for several models that do not have "almost circular" local interactions. The table includes the coordination number $z$, the critical point $p_{\mathrm{c}}$, the correction amplitudes $b_{\mathrm{i}}$ and $b_{1}$, and the amplitude $b_{3}$ of the $L^{-3}$ correction term. These correction amplitudes are to be compared with those for the "almost circular" models in Table II.

\begin{tabular}{|c|c|c|c|c|c|c|c|}
\hline$z$ & $p_{\mathrm{c}}$ & $L_{\min }$ & $b_{\mathrm{i}}$ & $b_{1}$ & $b_{3}$ & $\chi^{2}$ & $d_{\mathrm{f}}$ \\
\hline \multirow{2}{*}{$8+$} & $0.22149934(6)$ & 8 & $0.82(3)$ & $-0.364(9)$ & $-1.8(1)$ & 60.1 & 64 \\
\hline & $0.22149937(6)$ & 9 & $0.77(4)$ & $-0.35(1)$ & $-1.7(2)$ & 57 & 63 \\
\hline \multirow{2}{*}{$12 \times$} & $0.13805337(9)$ & 11 & $4.9(2)$ & $-0.79(4)$ & $-13.1(6)$ & 49 & 50 \\
\hline & $0.13805340(9)$ & 12 & $4.8(2)$ & $-0.75(5)$ & $-12(1)$ & 47.6 & 49 \\
\hline \multirow{2}{*}{$16 \times$} & $0.10321771(9)$ & 9 & $3.9(1)$ & $-0.70(3)$ & $-9.8(4)$ & 40 & 48 \\
\hline & $0.10321772(9)$ & 10 & $3.9(1)$ & $-0.69(4)$ & $-9.6(5)$ & 39.6 & 47 \\
\hline \multirow{2}{*}{$20+$} & $0.07831101(9)$ & 11 & $6.0(2)$ & $-1.38(5)$ & $-17.4(7)$ & 39.4 & 44 \\
\hline & $0.07831101(9)$ & 12 & $6.0(2)$ & $-1.39(6)$ & $-17.4(9)$ & 36.1 & 40 \\
\hline
\end{tabular}


Y.O. acknowledges the hospitality of the Lorentz Institute of the Leiden University, and H.B. thanks the University of Science and Technology of China in Hefei for hospitality extended to him.

\section{APPENDIX: ANALYSIS OF THE CORRECTION AMPLITUDES}

\section{Wrapping probabilities}

a. Almost spherical interaction regions

The Monte Carlo data for the wrapping probabilities were subjected to least-squares fits according to Eq. (39). The results for the amplitudes of the correction terms are listed in Tables II, III, and IV. The residual $\chi^{2}$, compared with the number of degrees of freedom, provides an indication of the reliability of the fits. One-sigma error margins are quoted, and they assume the validity and completeness of Eq. (39).

The data in Tables II, III, and IV show that the amplitudes $b_{\mathrm{i}}$ and $b_{1}$ of the three wrapping probabilities become very small between $z=4$ and $z=8$. This is, for example, also visible in Fig. 1. All three amplitudes grow significantly in absolute value with $z>8$, in accordance with the interpretation of $z$ as a measure of the distance to the short-range fixed point. An analysis of $b_{\mathrm{i}}(z)$ and $b_{1}(z)$ reveals a linear dependence on $z^{2}$ for systems with "almost circular" interaction regions. This behavior was illustrated in Fig. 3 .

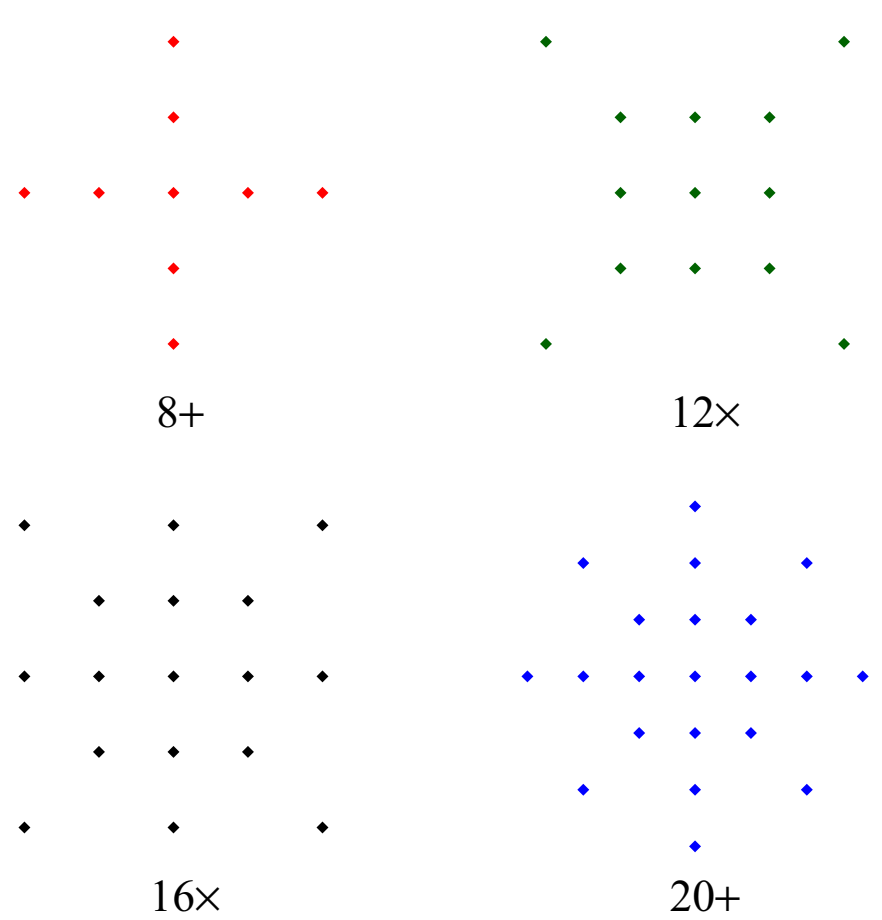

FIG. 14. Positions of the neighbors interacting with the central site. These four subfigures define models that disobey the condition that the interacting neighbors fill a circle.
TABLE VI. Finite-size scaling analysis of $R_{\mathrm{e}}$ for several models that do not have "almost circular" local interactions. The coordination number $z$, critical point $p_{\mathrm{c}}$, degenerate irrelevant field amplitudes $b_{\mathrm{i}}$ and $b_{1}$, amplitude $b_{3}$ of $L^{-3}$ correction term are listed.

\begin{tabular}{llcccccc}
\hline \hline$z$ & \multicolumn{1}{c}{$p_{\mathrm{c}}$} & $L_{\min }$ & \multicolumn{1}{c}{$b_{\mathrm{i}}$} & \multicolumn{1}{c}{$b_{\mathrm{l}}$} & $b_{3}$ & $\chi^{2}$ & $d_{\mathrm{f}}$ \\
\hline \multirow{2}{*}{$8+$} & $0.22149959(7)$ & 11 & $0.78(9)$ & $-0.41(3)$ & $2.4(4)$ & 62.9 & 57 \\
& $0.22149957(7)$ & 12 & $0.9(1)$ & $-0.43(3)$ & $2.0(4)$ & 61 & 56 \\
\multirow{4}{*}{$12 \times$} & $0.1380535(1)$ & 11 & $1.2(1)$ & $-1.02(3)$ & $-1.5(5)$ & 69.4 & 79 \\
& $0.1380537(1)$ & 12 & $0.7(2)$ & $-0.89(6)$ & $1(1)$ & 61.8 & 78 \\
\multirow{4}{*}{$20+$} & $0.1032180(1)$ & 12 & $1.3(2)$ & $-0.96(6)$ & $-1.1(9)$ & 48.4 & 43 \\
& $0.1032179(1)$ & 14 & $1.6(3)$ & $-1.04(8)$ & $-3(1)$ & 45.5 & 40 \\
& $0.07831096(9)$ & 11 & $3.1(2)$ & $-1.57(5)$ & $-2.4(8)$ & 47.6 & 44 \\
& $0.07831095(9)$ & 12 & $3.2(2)$ & $-1.59(6)$ & $-2.8(9)$ & 46.7 & 42 \\
\hline \hline
\end{tabular}

\section{b. Range dependence and fourfold symmetry perturbation}

While the local interactions assume an "almost circular" form for the large- $z$ models investigated thus far, the deviations from isotropy are obvious for small $z$. To study the possible effects of these fourfold deviations from circular symmetry on the correction amplitudes, we introduce a few models that do not obey the rule that the set of equivalent neighbors fills a circle with a given range $r$. Analysis of these models could possibly also distinguish between the effects of the coordination number $z$ and the distances to these neighbors. We refer to the newly chosen models as the $8+$ model, the $12 \times$ model, the $16 \times$ model, and the $20+$ model. The " $x$ " and "+" symbols show the orientation of the fourfold perturbation. These models are defined in Fig. 14. We do not consider the " $4 \times$ " model with interacting neighbors at $(x, y)=( \pm 1, \pm 1)$, which decouples into two disjoint percolation models, and thus belongs to a different category.

Monte Carlo simulations, and numerical analyses of the wrapping probabilities were performed, similar to those of the "almost circular" cases. The results are listed in Tables VVII. We found that the correction amplitudes did not fit in the simple picture of Fig. 3, i.e., neither $b_{\mathrm{i}}$ nor $b_{1}$ can be simply described by a quadratic function of $z$. To allow for the distances spanned by the $z$ interactions, we considered the dependencies of the amplitudes on the sums $\sum r_{i}^{k} z^{j}$, where $r_{i}$ is the $i$ th interacting neighbor, for several $k$ and $j$. As shown in Figs. 15, the amplitudes $b_{\mathrm{i}}$ and $b_{1}$ in $R_{\mathrm{b}}$ and $R_{\mathrm{e}}$ behave almost linearly as a function of $\left(\sum r_{i}^{2} / z\right)^{2}$.

TABLE VII. Analysis of the correction amplitudes in $R_{1}$ for several models that do not have "almost circular" local interactions.

\begin{tabular}{llcccccc}
\hline \hline$z$ & \multicolumn{1}{c}{$p_{\mathrm{c}}$} & $L_{\min }$ & \multicolumn{1}{c}{$b_{\mathrm{i}}$} & $b_{\mathrm{l}}$ & \multicolumn{1}{c}{$b_{3}$} & $\chi^{2}$ & $d_{\mathrm{f}}$ \\
\hline \multirow{2}{*}{$8+$} & 0.2214995 & 11 & $0.11(4)$ & $-0.06(1)$ & $1.6(2)$ & 60 & 58 \\
& 0.2214995 & 12 & $0.14(4)$ & $-0.07(1)$ & $1.5(2)$ & 57.5 & 57 \\
\multirow{4}{*}{$12 \times$} & 0.13805374 & 11 & $-1.80(5)$ & $-0.14(1)$ & $5.4(2)$ & 67 & 55 \\
& 0.13805374 & 12 & $-1.87(7)$ & $-0.12(2)$ & $5.9(4)$ & 65.1 & 54 \\
\multirow{4}{*}{$2 \times+$} & 0.1032177 & 14 & $-1.1(1)$ & $-0.17(3)$ & $3.3(6)$ & 37 & 37 \\
& 0.1032177 & 16 & $-0.9(2)$ & $-0.22(4)$ & $1.9(9)$ & 31.3 & 34 \\
& 0.0783110 & 11 & $-1.40(7)$ & $-0.10(2)$ & $7.3(3)$ & 47.3 & 46 \\
& 0.0783110 & 12 & $-1.41(9)$ & $-0.10(2)$ & $7.4(4)$ & 45.9 & 42 \\
\hline \hline
\end{tabular}




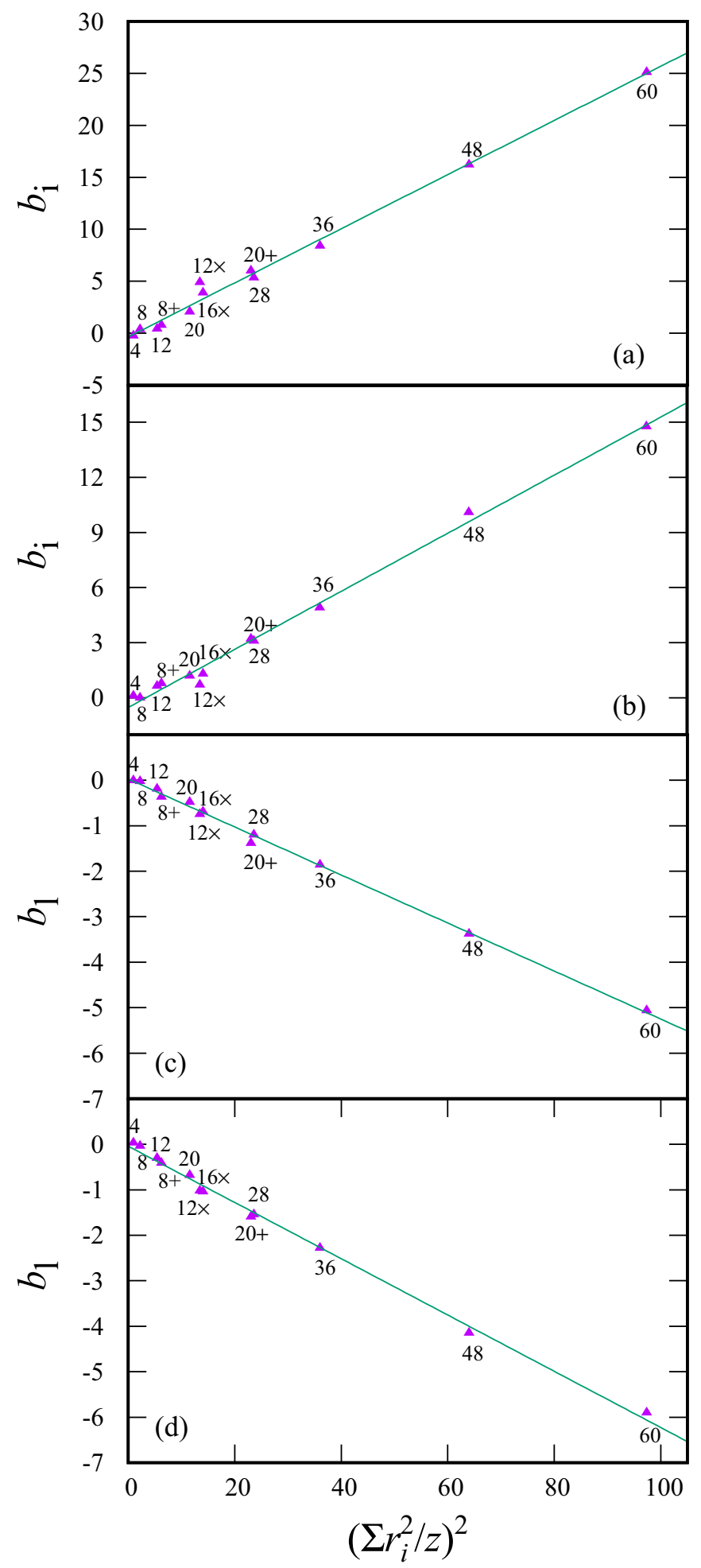

FIG. 15. Correction amplitudes in $R_{\mathrm{b}}$ and $R_{\mathrm{e}}$ vs. the square of the sum $\Sigma_{j} r_{j}^{2} / z$. (a) $b_{\mathrm{i}}$ in $R_{\mathrm{b}}$; (b) $b_{\mathrm{i}}$ in $R_{\mathrm{e}}$; (c) $b_{1}$ in $R_{\mathrm{b}}$; (d) $b_{1}$ in $R_{\mathrm{e}}$. The data points in the figures are labeled with the corresponding coordination numbers $z$.

We have attempted to further improve the description of the dependence of $b_{\mathrm{i}}$ and $b_{1}$ on the local interaction neighborhood, by including a few more sums over the interacting neighbors, involving polar coordinates $r_{i}$ and the angle $\phi_{i}$ with respect to
TABLE VIII. Finite-size amplitudes of the critical Binder ratio $Q$, for several values of the coordination number $z$. For finite $z$, fits were applied according to Eq. (28) with an additional term $c_{1}(p-$ $\left.p_{c}\right) L^{y_{t}-2}$, which accounts for the dependence of the $p$-dependent term on the irrelevant field. For $z>4, Q$ was fixed at the universal value determined for the 4-neighbor model, and the percolation threshold $p_{\mathrm{c}}$ was fixed at the value found earlier from the wrapping probabilities. The errors between parentheses are shown as onesigma margins in the last decimal place listed. Actual uncertainties can be a few times larger after taking into account the errors in $p_{\mathrm{c}}$ and $Q$. The exponents $y_{t}$ and $y_{h}$ were fixed at their exactly known values in all cases. For the complete graph (cg) data, the value of $Q$ was left free in the fit formula, while the percolation threshold was fixed at the integrated probability $p_{\mathrm{i}, \mathrm{c}}=1$. Three finite-size correction terms, with fixed exponents $-2 / 3,-4 / 3$, and -2 were included for the complete graph case. The respective amplitudes are given in the last three columns.

\begin{tabular}{rrccrrr}
\hline \hline$z$ & $L_{\min }$ & $p_{\mathrm{c}}$ & $Q$ & \multicolumn{1}{c}{$b_{\mathrm{i}}$} & \multicolumn{1}{c}{$b_{1}$} & \multicolumn{1}{c}{$b_{3}$} \\
\hline \multirow{4}{*}{4} & 10 & $1 / 2$ & $0.87057(1)$ & $3.68(15)$ & $1.25(12)$ & $-4.1(3)$ \\
& 11 & & $0.87057(1)$ & $3.79(19)$ & $1.34(14)$ & $-4.3(4)$ \\
& 13 & 0.2503685 & 0.87057 & $0.37(15)$ & $-1.0(2)$ & $0.9(4)$ \\
& 14 & & & $0.42(17)$ & $-0.9(2)$ & $0.7(5)$ \\
& 16 & 0.1522203 & 0.87057 & $-8.5(2)$ & $-9.7(4)$ & $18.2(8)$ \\
12 & 18 & & & $-8.4(2)$ & $-9.4(5)$ & $17.6(10)$ \\
& 18 & 0.0841509 & 0.87057 & $-17.9(3)$ & $-22(1)$ & $42(2)$ \\
20 & 20 & & & $-17.8(4)$ & $-22(1)$ & $42(3)$ \\
& 20 & 0.0558493 & 0.87057 & $-29.9(2)$ & $-48.8(5)$ & $88(1)$ \\
28 & 22 & & & $-29.9(2)$ & $-49.1(7)$ & $89(1)$ \\
& & & & & & \\
36 & 22 & 0.04169608 & 0.87057 & $-35.8(2)$ & $-70.9(8)$ & $125(2)$ \\
& 24 & & & $-36.0(2)$ & $-69.9(9)$ & $124(2)$ \\
48 & 24 & 0.02974268 & 0.87057 & $-37.2(2)$ & $-121(1)$ & $206(1)$ \\
& 28 & & & $-37.7(3)$ & $-119(1)$ & $203(2)$ \\
60 & 24 & 0.0230119 & 0.87057 & $-31.1(10)$ & $-184(5)$ & $307(9)$ \\
& 28 & & & $-29.9(14)$ & $-190(7)$ & $316(12)$ \\
& 19 & 1 & $0.38127(5)$ & $0.158(2)$ & $0.13(3)$ & $0.25(10)$ \\
$\operatorname{cg}$ & 20 & & $0.38125(6)$ & $0.159(2)$ & $0.12(3)$ & $0.32(11)$ \\
\hline \hline
\end{tabular}

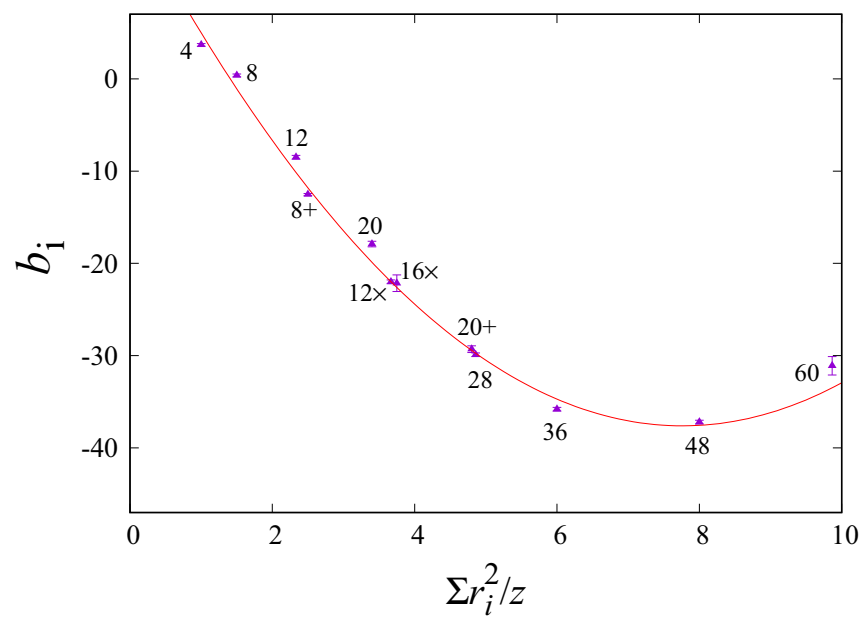

FIG. 16. Correction to scaling amplitude $b_{\mathrm{i}}$ in the Binder ratio $Q$ vs. $\sum_{i} r_{i}^{2} / z$. These data, which are taken from Table VIII, include the models that do not have an almost spherical set of interacting neighbors. These data are approximately described by the parabola shown in the figure. 
a nearest neighbor. While a fit of the data by

$$
\begin{aligned}
b_{\mathrm{i} / \mathrm{l}}= & c_{0}\left(\sum_{i} r_{i} / z\right)^{2}+c_{1} \sum_{i} r_{i}^{2}+c_{2} \sum_{i} r_{i} \cos \left(4 \phi_{i}\right) \\
& +c_{3}\left[\sum_{i} r_{i} \cos \left(4 \phi_{i}\right)\right]^{2}+c_{4} \sum_{i} r_{i}^{2} / z \\
& +c_{5}\left(\sum_{i} r_{i}^{2} / z\right)^{2}+c_{6} \sum_{i} r_{i}^{4}
\end{aligned}
$$

did reduce the residual $\chi^{2}$, we doubt the physical meaning of the resulting parameters, which were seen to depend considerably on variations of the fit formula. No clear dependence of the amplitudes on the orientation of the fourfold anisotropy, i.e., contributions due to a term with $\cos \left(4 \phi_{i}\right)$, was found.

\section{Correction amplitudes of the Binder ratio}

Least-squares fits according to Eq. (27) to the finite-size data for the Binder ratio gave clear signs of the presence of the term $b_{4} L^{y_{t}-2 y_{h}}$, i.e., the existence of a quadratic contribution of the physical magnetic field to the temperature field. Results these fits are listed in Table VIII. In particular the data for $b_{\mathrm{i}}$, which are also shown in Fig. 16, display a different behavior than, for instance, the approximately linear dependence on $z^{2}$ as seen in Fig. 3. This suggests that the finite-size-scaling functions of the magnetization moments depend nonlinearly on the irrelevant field associated with the interaction range. In analogy with the wrapping probabilities, a comparison between Figs. 6 and 16 shows that the replacement of $z$ by the quantity $\sum_{i} r_{i}^{2} / z$ serves well to describe the influence of the neighbor distances on the correction amplitudes.
[1] M. E. Fisher, S.-K. Ma, and B. G. Nickel, Phys. Rev. Lett. 29, 917 (1972).

[2] J. Sak, Phys. Rev. B 8, 281 (1973).

[3] E. Luijten and H. W. J. Blöte, Phys. Rev. B 56, 8945 (1997); Phys. Rev. Lett. 89, 025703 (2002).

[4] E. Luijten, H. W. J. Blöte, and K. Binder, Phys. Rev. E 54, 4626 (1996); 56, 6540 (1997).

[5] X. F. Qian, Y. Deng, Y. Liu, W.-A. Guo, and H. W. J. Blöte, Phys. Rev. E 94, 052103 (2016).

[6] B. Nienhuis, A. N. Berker, E. K. Riedel, and M. Schick, Phys. Rev. Lett. 43, 737 (1979).

[7] J. W. Essam in Phase Transitions and Critical Phenomena, edited by C. Domb and M. S. Green (Academic Press, New York, 1976), Vol. 2.

[8] D. Stauffer, Phys. Rep. 54, 1 (1979).

[9] P. J. Reynolds, H. E. Stanley, and W. Klein, Phys. Rev. B 21, 1223 (1980).

[10] R. B. Potts, Proc. Cambridge Philos. Soc. 48, 106 (1952).

[11] P. W. Kasteleyn and C. M. Fortuin, J. Phys. Soc. Jpn. 26 (Suppl.), 11 (1969); C. M. Fortuin and P. W. Kasteleyn, Physica (Amsterdam) 57, 536 (1972).

[12] E. Luijten and H. W. J. Blöte, Int. J. Mod. Phys. C 6, 359 (1995).

[13] K. Binder, Z. Phys. B 43, 119 (1981).

[14] B. Nienhuis, in Phase Transitions and Critical Phenomena, edited by C. Domb and J. L. Lebowitz (Academic Press, New York, 1987), Vol. 11. In Eq. (4.26) of this work, +1 has to be replaced by +2 .

[15] M. E. Fisher, in Critical Phenomena, Proc. 51st Enrico Fermi Summer School, Varenna, edited by M. S. Green (Academic Press, New York, 1972).

[16] M. Suzuki, Progr. Theor. Phys. 58, 1142 (1977).

[17] M. P. Nightingale in Finite-size Scaling and Numerical Simulation of Statistical Systems, edited by V. Privman (World Scientific, Singapore, 1990).
[18] Th. Niemeijer and J. M. J. van Leeuwen, in Phase Transitions and Critical Phenomena, edited by C. Domb and M. S. Green (Academic Press, New York, 1976), Vol. 6.

[19] X. F. Qian, Y. Deng, and H. W. J. Blöte, Phys. Rev. E 72, 056132 (2005).

[20] F. J. Wegner, in Phase Transitions and Critical Phenomena, edited by C. Domb and M. S. Green (Academic Press, New York, 1976), Vol. 6.

[21] L. Mittag and M. J. Stephen, J. Phys. A 7, L109 (1974).

[22] F. Y. Wu, Rev. Mod. Phys. 54, 235 (1982).

[23] A. Drory, Phys. Rev. E 54, 6003 (1996).

[24] B. Bollobás, G. Grimmett, and S. Janson, Probab. Th. Rel. Fields 104, 283 (1996).

[25] R. Kenna and B. Berche, J. Phys. A 50, 235001 (2017).

[26] K. Binder, M. Nauenberg, V. Privman, and A. P. Young, Phys. Rev. B 31, 1498 (1985).

[27] E. Brézin and J. Zinn-Justin, Nucl. Phys. B 257, 867 (1985).

[28] H. Hu, H. W. J. Blöte, R. M. Ziff, and Y. Deng, Phys. Rev. E 90, 042106 (2014).

[29] H. Hu, H. W. J. Blöte, and Y. Deng, J. Phys. A 45, 494006 (2012).

[30] H. T. Pinson, J. Stat. Phys. 75, 1167 (1994).

[31] R. M. Ziff, C. D. Lorenz, and P. Kleban, Physica A 266, 17 (1999).

[32] M. E. J. Newman and R. M. Ziff, Phys. Rev. Lett. 85, 4104 (2000)

[33] X. F. Qian, Y. Deng, and H. W. J. Blöte, Phys. Rev. B 71, 144303 (2005).

[34] Y. Deng and H. W. J. Blöte, Phys. Rev. E 71, 016117 (2005).

[35] J. L. Cardy, in Phase Transitions and Critical Phenomena, edited by C. Domb and J. L. Lebowitz (Academic Press, London, 1987), Vol. 11, p. 55, and references therein.

[36] W. Huang, P. Hou, J. Wang, R. M. Ziff, and Y. Deng, Phys. Rev. E 97, 022107 (2018). 